\title{
What triggers transient AIDS in the acute phase of HIV infection and chronic AIDS at the end of the incubation period?
}

\author{
A model analysis of HIV infection from the acute phase to the chronic \\ AIDS stage
}

IVAN KRAMER*

Physics Department, University of Maryland Baltimore County, 1000 Hilltop Circle, Catonsville, MD 21250, USA

(Received 26 April 2006; revised 11 July 2006; in final form 28 February 2007)

\begin{abstract}
Novel dynamical models are introduced demonstrating that the T helper cell (THC) density drops in the acute infection phase of HIV infection, sometimes causing transient AIDS, and at the end of the incubation period causing chronic AIDS have a common dynamical cause. The immune system's inability to produce enough uninfected THCs to replace the infected ones it is destroying causes a drop in the THC density at any stage of HIV infection. Increases in viral infectivity, probably caused by random mutation of HIV, are shown to drive the progression of the infection. The minimum incubation period for the long term non-progressors (LTNPs) was calculated from a novel physical model: $0.3 \%$ of infecteds have incubation periods of 23.1 years or more, and there is no biomedical difference between LTNPs and progressors. Chronic AIDS is shown to result from three random transitions linking four clinically-distinct stages of HIV infection following seroconversion.
\end{abstract}

Keywords: HIV; AIDS; Infectivity; Incubation period; CD4+ cells; Co-receptor blockers

\section{Introduction: the stages of HIV infection}

Novel dynamical models are utilized here to answer some of the outstanding, perplexing questions about HIV infection. Dynamical modeling is a powerful technique that is frequently indispensable in synthesizing disparate data to answer fundamental questions about an infection or medical condition.

The primary targets of HIV-1 virions are infectable CD4+ cells of the immune system, including T helper cells (THCs) and a subpopulation of natural Killer cells. By penetrating a cell's membrane and seizing control of its reproductive apparatus, a virion causes copies of itself to be reproduced when the cell is stimulated. In addition to the CD4+ molecule, a target cell must have a co-receptor molecule, e.g. the CCR5 or CXCR4 cell surface receptor, in order for a virion to be able to bind to the cell and infect it. Indeed, about $1 \%$ of Caucasians

*Email: kramer@umbc.edu

Computational and Mathematical Methods in Medicine 
lack co-receptor molecules, and, therefore, are completely immune to becoming HIVinfected. Mutations of the virus occur when errors are made in the transcription of viral RNA into DNA following HIV penetration of a target cell.

The immune system's job is to minimize the ability of virions to infect target cells, minimize virion production in infected CD4 + cells, and clear virions and infected cells from the host.

Once a host is exposed to enough virions to generate an anti-body response (seroconversion), experience shows that the host is probably irreversibly HIV-infected. Experiment shows that an HIV-infected immune system, even aided by highly active antiretroviral therapy (HAART), treated with interleukin-2 (IL-2), or stimulated with experimental vaccines, cannot clear the infection.

All HIV infections pass through an acute infection stage, which may be asymptomatic, in which the viral load grows exponentially and a sizable fraction (sometimes over 50\%) of a patient's THCs become infected before the immune system reacts to reverse this situation. A characteristic of HIV infection during the acute phase is the decline in the total THC density, sometimes to clinically dangerous levels, and its subsequent rebound after the immune system's reactions to the challenge develop. What is not commonly known is that immunosuppression during the acute infection stage can be so severe that the host (infected) can develop AIDS for a brief period of time, clinically identical (except for its duration) to the chronic AIDS state that signals the end of the incubation period. Thus, it is possible for a transient AIDS state to develop in the acute phase of the infection. This fact alone is incompatible with the antigenic diversity model of the cause of AIDS in HIV infection.

According to the modeling presented here, when the rate of destruction of infected THCs exceeds the maximum production rate of uninfected THCs, the total THC density must drop in any stage of HIV infection. Modeling of transient AIDS state data leads to the prediction that well over $10 \%$ of the total THCs of these patients became infected at some time in the acute infection phase, a percentage that agrees with experimental measurements in the literature.

After seroconversion, which generally occurs about a month after inoculation with HIV, the infection settles down to a quasi-static steady-state phase (the incubation period) during which the viral load and the uninfected and infected CD4 + cell densities can be considered quasi-constants in time, changing slowly on a day-to-day basis. Modeling the incubation period distribution curve (IPDC) leads to the prediction that there are four clinical stages to HIV infection following seroconversion, and the development of chronic AIDS is not the result of a single random transition, but the result of an ordered chain of three random transitions. This prediction is completely compatible with the World Health Organization's staging of HIV infection that identified four clinically distinct stages of HIV infection following the acute infection phase. The modeling here demonstrates that the evolution of HIV infection is driven by the relentless increase in viral infectivity, defined as the probability that an uninfected target CD4 + cell will become infected after one encounter with a virulent virion. This definition of viral infectivity is proportional to a measurable parameter that will be introduced later in this paper. The evolution of the value of the viral infectivity, in turn, is driven by random mutations of the virus.

What is the model's answer to the question "what determines the great variation in incubation period leading to chronic AIDS?"

The modeling here demonstrates that the fraction of a cohort in any stage of HIV infection that makes the transition to the next stage per unit time is 0.2513 per year. Expressed another way, half of those in a given stage of HIV infection will make the transition to the next stage 
in 2.76 years. Thus, only $0.3 \%$ of infecteds will be found in stage 1 of HIV infection 23.1 years after seroconversion - these are clearly the long-term nonprogressors (LTNPs) to HIV infection. However, since the mutation rate, and, therefore, the rate of change of the viral infectivity, for any individual is probably unconnected to any biomedical parameter or profile of the infected, it is probably impossible to tell the difference between the LTNPs and the progressors, and, indeed, no such difference has been found to date. In the novel model presented here, the LTNPs are simply those whose viral infectivities grow so slowly in time that they remain in stage 1 of the infection for unusually long periods of time. LTNPs are simply lucky that random mutation of their viral profiles has not led to a rapid (or any!) increase in the values of their viral infectivities. By contrast, an infected whose viral mutations lead to a rapid increase in viral infectivity rapidly progresses to AIDS (stage 4 of the disease); the incubation period for such an infected is, therefore, very short.

If these modeling results are correct, then there is no unique, special anti-viral substance secreted by the CD8 $+\mathrm{T}$ cells of LTNPs that accounts for their unusually long incubation periods. Indeed, to date, no such substance has been found although researchers have sought this substance for many years.

In the 2-year period leading up to the onset of chronic AIDS, the infected's viral load begins to rise. The quasi-static, steady-state phase begins to end when the infected THC density and viral load reach critical values, after which the THC density drops precipitously by one or more orders of magnitude; a new steady-state is subsequently reached by which time the patient has developed AIDS. The drop in the total THC density in the acute infection phase and at the end of the incubation period leading to chronic AIDS will be shown to have a common dynamical cause, namely, the inability of the immune system to replace all the infected THCs it is destroying with uninfected ones.

The important dynamical difference between the transient AIDS state in the acute infection stage and the chronic AIDS state that signals the end of the incubation period is the value of the viral infectivity; the modeling here demonstrates that, following the acute phase, the viral infectivity of target CD4 + cells grows by a factor of 10-100 causing chronic AIDS to develop. The model presented here allows the relative value of the viral infectivity to be computed in any stage of HIV infection, as will be shown in a specific case study at the end of this paper.

From the modeling results in this paper, the most direct way to control the value of the viral infectivity and, thereby, prevent the development of chronic AIDS, is to discover effective, tolerable, co-receptor fusion blockers. Such blockers would be able to limit the number of THCs that are infected so that the infected THC destruction rate would always be less than the maximum value of the uninfected THC production rate.

However, recent experiments on HIV-infected patients using an enzymatically-modified serum vitamin $\mathrm{D}_{3}$-binding protein, called Gc macrophage activating factor (GcMAF), stimulated macrophage action against HIV and eradicated all traces of viral antigens in the patients' blood for 2 years after the completion of therapy. Thus, hope of finding a way to strengthen the immune response against HIV infection so that the fatal nature of the disease is eliminated should not be abandoned.

\section{Dynamically describing HIV infection}

To dynamically describe HIV infection in all of its stages, it is necessary to consider a set of model equations that phenomenologically simulates the time-dependent interaction between the virus and the immune system. 
The target cells of HIV-1 are infectable CD4 + cells of the immune system itself, such as THCs and a CD4+ subpopulation of natural killer cells.

As with any antigen infected cell, the immune system goes about destroying infected $\mathrm{CD} 4+$ cells at a certain rate but, to maintain the viability of the immune system, replaces these cells with uninfected ones at the same rate in order to keep the total CD4 + cell density constant. As long as the production rate of uninfected THCs can keep up with the destruction rate of infected THCs, the total THC density will remain constant. However, as the infectivity of THCs increases, the number of THCs that are infected increases, the rate of destruction of infected THCs must, thereby, increase, and uninfected THC production must increase to keep the total THC density constant. There comes a point, however, when the uninfected THC production of the immune system cannot keep up with the rate the immune system is destroying infected THCs, and the total THC density must inevitably drop. This dynamical situation constitutes the trigger that causes the decline in the total THC density leading to AIDS.

The key immunological parameters that characterize the state of the infection are all time dependent in general. The uninfected $\mathrm{CD} 4+$ cell density in the peripheral blood at time $t$ will be denoted by $T(t)$, the infected $\mathrm{CD} 4+$ cell density will be denoted by $T *(t)$, and the virion density (viral load) will be denoted by $V(t)$. The total CD4 + cell density, denoted by $T^{+}(t)$, is the sum of the infected and uninfected CD4+ cell densities so that $T^{+}(t)=T(t)$ $+T^{*}(t)$. The values of these densities are related to each other through model equations.

If the elapsed time after inoculation with a dose of HIV virions is denoted by $t$, then the simulation of the dynamics of HIV infection in the host's peripheral blood assumed in this paper is represented by the following set of coupled, first-order, non-linear differential equations:

$$
\begin{gathered}
\frac{\mathrm{d} V(t)}{\mathrm{d} t}=p(t) T^{*}(t)-d(t) V(t) \\
\frac{\mathrm{d} T^{*}(t)}{\mathrm{d} t}=p^{*}(t) T(t) V(t)-d^{*}(t) T^{*}(t) \\
\frac{\mathrm{d} T(t)}{\mathrm{d} t}=g(t)-p^{*}(t) T(t) V(t) \\
\frac{\mathrm{d} T^{+}(t)}{\mathrm{d} t}=-d^{*}(t) T^{*}(t)+g(t)
\end{gathered}
$$

where $p(t), d(t), p^{*}(t), d^{*}(t)$ and $g(t)$ are model parameter input functions. The non-linearity of this set of coupled equations stems from the $T(t) V(t)$ term on the right hand sides of equations (1b) and (1c).

Subtracting the sum of equations (1b) and (c) from (1d) and integrating the result gives

$$
T^{+}(t)=T(t)+T^{*}(t),
$$

as it must.

Thus, the viral clearance rate parameter $d(t)$ determines the average instantaneous rate at which the host's immune system clears virions and the infected $C D 4+$ cell clearance rate 
parameter $d *(t)$ determines the average instantaneous rate at which infected CD4 + cells are cleared. The impact of CD8+ T cells on HIV infection is included in the $d *(t)$ term in (1b). Although a time-dependent $d *(t)$ is allowed in this modeling, this parameter can be considered to be an approximate constant during any stage of HIV infection in which the viral load and infected THC density are not changing.

The viral production rate parameter $p(t)$ is the overall average rate at which virions are produced per infected CD4 + cell, and the infectivity rate parameter $p *(t)$ is a measure of HIV's ability to infect uninfected CD4+ cells on the average. The infectivity rate parameter $p *(t)$ is measurable in principle and is proportional to the viral infectivity, denoted by $\inf (t)$ and defined as the probability that an uninfected target $\mathrm{CD} 4+$ cell will become infected after one encounter with a virulent virion. Because the other factors in the definition of $p *(t)$ are constants for any infected, measurement of $p *(t)$ for any two different times $t_{1}$ and $t_{2}$ gives $\left(p^{*}\left(t_{2}\right)\right) /\left(p^{*}\left(t_{2}\right)\right)=\inf \left(t_{2}\right) / \inf \left(t_{1}\right)$. This last relation will be used in a later section of this paper.

The uninfected THC regeneration rate parameter $g(t)$ in (1c) and (1d) is simply the net average rate uninfected THCs are produced per unit volume of peripheral blood. The thymus plays an important role in contributing to the value of this parameter.

The absence of a term like $-d_{T}(t) T(t)$ from the right hand side of (1c), where $d_{T}(t)$ is the natural uninfected THC destruction function in the absence of disease, is justified by defining $g(t)$ in such a way as to include this term.

Because of equations (1b), (1c) and (1e), the first term on the right-hand side of equation (1d) describes the rate of loss of infected CD4 + cells while the second term on the righthand side of equation (1d) describes the rate of production of uninfected CD4 + cells. Note that the conversion of uninfected CD4 + cells into an equal number of infected CD4 + cells does not change the value of the total CD $4+$ cell density $T^{+}$. The production parameter $g(t)$ is the net rate at which new uninfected $\mathrm{CD} 4+$ cells are produced per volume of peripheral blood. If the total CD4 + cell density $T^{+}$is a constant during a period of the chronic HIV infection, then equation (1d) gives $g(t)=d^{*}(t) T^{*}(t)$ so that the immune system is replacing all the infected CD4 + cells that it is destroying with an equal number of uninfected ones.

Now the functions $T(t), T^{*}(t)$ and $T^{+}(t)$ that appear in equations (1a)-(1e) are sums over all HIV-infectable CD4 + cells in the host and, therefore are sums over compartments of different cell types, such as THCs, a subset of NK cells, and primary macrophages [1-3].

The model presented here is a generalization of the one constructed by Perelson et al. which assumed that all four of the model parameters $p, p^{*}, d$ and $d^{*}$ were constants, the target cells of HIV were exclusively THCs, the infected THC density $T *$ was very much smaller than uninfected THC density $T$, and the total THC density $T^{+}$was a constant [4]. The Perelson model was successfully used to produce the first estimates of the values of the destruction rates $d$ and $d *$ and led to the first estimates of the turnover rates of THCs and virions during the infection. Clearly, equations (1a)-(1e) constitute a phenomenological model that monitors the gross results of a highly complex antigen-immune system interaction. Since the THC density is the dominant compartment in HIV infection, the densities in all the other HIV infectable compartments are very small with respect to this one. Thus, the Perelson model can be regarded as a good approximation to HIV infection, with the other compartments viewed as perturbations on the results stemming from this approximation. From a numerical standpoint, applying equations (1a)-(1e) to the THC compartment leads to results that are in good agreement with experiment. However, from an immunological standpoint, the HIV-infected CD4+ subset of NK cells and the latently infected long-lived memory $\mathrm{T}$ cells cannot be ignored because these infected cells make clearance of the infection impossible [5]. 
With this understanding, equations (1a)-(1e) will be applied to the THC compartment in the simulations to follow in this paper. These simplified simulations of HIV infection will be shown to give good numerical agreement with disparate data on HIV infection in the literature and lead to a deeper understanding of HIV infection and AIDS. This model was also used to help explain why preventative vaccines, therapeutic vaccines, and IL-2 therapy are not working against HIV infection [5].

\section{Saturation or the steady-state solution}

Useful information can be obtained about the infection challenge by investigating the steadystate equilibrium that results from the saturation of the model equations. If all the quantities in equations (1a)-(1d) saturate and become constants, then the infection will be said to have reached an equilibrium state at a time denoted by $t_{\mathrm{e}}$. Setting equations (1a)-(1c) equal to zero, the steady-state values of $V, T, T *$ and $T^{+}$become the following functions of the model parameters:

$$
\begin{gathered}
V\left(t_{\mathrm{e}}\right)=\frac{p\left(t_{\mathrm{e}}\right) g\left(t_{\mathrm{e}}\right)}{d\left(t_{\mathrm{e}}\right) d^{*}\left(t_{\mathrm{e}}\right)}, \\
T^{*}\left(t_{\mathrm{e}}\right)=\frac{g\left(t_{\mathrm{e}}\right)}{d^{*}\left(t_{\mathrm{e}}\right)}, \\
T\left(t_{\mathrm{e}}\right)=\frac{\mathrm{d}\left(t_{\mathrm{e}}\right) d^{*}\left(t_{\mathrm{e}}\right)}{p\left(t_{\mathrm{e}}\right) p^{*}\left(t_{\mathrm{e}}\right)}, \\
T^{+}\left(t_{\mathrm{e}}\right)=\frac{g\left(t_{\mathrm{e}}\right)}{d^{*}\left(t_{\mathrm{e}}\right)}+\frac{d\left(t_{\mathrm{e}}\right) d^{*}\left(t_{\mathrm{e}}\right)}{p\left(t_{\mathrm{e}}\right) p^{*}\left(t_{\mathrm{e}}\right)} .
\end{gathered}
$$

After seroconversion, the HIV infection settles down to a prolonged steady-state described by the functions in $(2 a)-(2 d)$. The viral set-point is given by equation (2a), the infected $\mathrm{CD} 4+$ cell density is given by $(2 \mathrm{~b})$, the uninfected $\mathrm{CD} 4+$ cell density is given by $(2 \mathrm{c})$, and the total CD4 + cell density is given by equation (2d). In fact, the model parameters in (2a)(2d) can be slowly varying functions of time so that the steady-state becomes a quasi-static one. Exactly what happens during this prolonged, quasi-static, steady-state to trigger the onset of the development of AIDS is one of the questions this paper will address and answer.

All of the tables and graphs that are presented in the following sections contain data that is either used to construct the model in this paper or to confirm it. To help distinguish between data and model calculations, all data in the tables and graphs are labeled as such.

\section{Constructing a chronic AIDS transition model}

Saksela et al. [6] conducted an extended study of 18 HIV-infected patients with initial THC densities in the normal range to determine the changes in immunological parameters that foreshadowed the subsequent development of AIDS. At the beginning of the study, no 
Table 1. Acute HIV infection data from Ref. [8]. All three patients had Pneumocystis carinii pneumonia at presentation. Patient 3 also developed oral candidosis on day-11.

\begin{tabular}{|c|c|c|c|c|c|}
\hline \multicolumn{6}{|c|}{ Patient $1[8]$} \\
\hline $\begin{array}{l}\text { Time } t(\mathrm{~d} \equiv \text { days }) \\
-90 \mathrm{~d} \text { (preinfection) }\end{array}$ & $\begin{array}{l}T^{+}(t)^{\dagger} \text { data }\left(\text { cells } / \mathrm{mm}^{3}\right) \\
920 \text { (baseline) }\end{array}$ & $\left\langle\mathrm{d} T^{+}(t) / \mathrm{d} t\right\rangle^{\ddagger}$ data $\left(\right.$ cells $\left./ \mathrm{mm}^{3}\right) / \mathrm{d}$ & $\langle T *(t)\rangle^{\natural}$ data $\left(\right.$ cells $\left./ \mathrm{mm}^{3}\right)$ & $\left\langle T^{+}(t)\right\rangle^{\S}\left(\right.$ cells $\left./ \mathrm{mm}^{3}\right)$ & $\langle T *(t)\rangle\left\langle\left\langle T^{+}(t)\right\rangle^{\|} \times 100 \%\right.$ \\
\hline-45 days & & 0 & 3.6 & 920 & $0.39 \%$ \\
\hline-22.5 days & & -14.2 & 32.0 & 600 & $5.33 \%$ \\
\hline 0 (presentation) & 280 & & & & \\
\hline 5 days & & -27.1 & 57.8 & 144.5 & $40.0 \%$ \\
\hline 10 days $(\mathrm{PCP})$ & 9.1 & 0 & 3.6 & 9.1 & $39.6 \%$ \\
\hline 30 days & 390 & & & & \\
\hline 180 days & 942 & & & & \\
\hline 4 years & 589 & & & & \\
\hline \multicolumn{6}{|c|}{ Patient 2 [8] } \\
\hline-60 days (preinfection) & 1216 (baseline) & & & & \\
\hline-30 days & & 0 & 3.6 & 1216 & $0.30 \%$ \\
\hline-15 days & & -29.7 & 63 & 770.5 & $8.18 \%$ \\
\hline 0 (presentation) & 325 & & & & \\
\hline 7 days & & -18.8 & 41.2 & 193.5 & $21.3 \%$ \\
\hline 14 days (PCP) & 62 & 0 & 3.6 & 62 & $5.8 \%$ \\
\hline 45 days & 210 & & & & \\
\hline 4 months & 590 & & & & \\
\hline 1 year & 715 & & & & \\
\hline 39 months & 523 & & & & \\
\hline \multicolumn{6}{|c|}{ Patient 3 [8] } \\
\hline-50 days (preinfection) & 895 (baseline) & & & & \\
\hline-25 days & & 0 & 3.6 & 895 & $0.40 \%$ \\
\hline-12.5 days & & -28.8 & 61.3 & 534.5 & $11.5 \%$ \\
\hline 0 (presentation) & 174 & & & & \\
\hline 4 days & & -11.4 & 26.4 & 128.5 & $20.5 \%$ \\
\hline 8days (PCP) & 83 & 0 & 3.6 & 83 & $4.34 \%$ \\
\hline 30 days & 343 & & & & \\
\hline 3 months & 687 & & & & \\
\hline 21 months & 416 & & & & \\
\hline 29 months & 494 & & & & \\
\hline
\end{tabular}

${ }^{\dagger}$ Total THC density data, $T^{+}(t)$

Average value of $\mathrm{d} T^{+}(t) / \mathrm{d} t$

Model computation of average infected THC density, $\langle T *(t)\rangle$

Average total THC density, $\left\langle T^{+}(t)\right\rangle$. 
detectable HIV-1 messenger ribonucleic acid (mRNA) expression was found in the peripheral blood mononuclear cells (PBMCs) of any of the subjects. Saksela et al. discovered that in the 2 year period leading up to the beginning of the spontaneous drop in the total THC density of the seven study subjects who developed AIDS, detectable levels of viral mRNA were found in these patients' PBMCs (table 1 in [6]).

A study of HIV-1 density in peripheral blood mononuclear cells (PBMC) and in plasma of 54 HIV-infected patients who were not receiving anti-viral therapy was conducted by David Ho et al. [7]. This study found that the viral density in patients with either AIDS related complex (ARC) or AIDS were much higher than in asymptomatic infecteds on average. Most interesting, however, was the finding that ARC and AIDS patients had virtually the same distribution of viral densities in either plasma or PBMC (figure 1 in [7]). One possibility suggested by this data is that the viral load at the beginning of the ARC stage and its steady-state value developed in the AIDS stage for every HIV infected patient are identical. This possibility will be explored in the AIDS transition model to be described below.

The simplest simulation of the above data on the transition to chronic AIDS is to assume that the infectivity rate parameter $p *(t)$ starts to increase due to HIV mutation, thereby increasing the infected THC density $T *(t)$ (equation (1b)). This increase in $T *(t)$ causes an increase in the viral load $V(t)$ [see equation (1a)]. At some point the second-term on the righthand side of (1c) exceeds the maximum value of the uninfected THC density regeneration rate $g(t)$ so that uninfected THC density $T(t)$ drops.

The model describing the development of AIDS to be constructed here is consistent with the data in Refs [6,7]. The following is a list of assumptions, consistent with the data in the literature, which will be used to construct the chronic AIDS model that follows.

\subsection{AIDS transition model assumptions and features}

(a) It will first be assumed that the state of the infection at the beginning and end of the THC density drop ending in chronic AIDS are quasi-static equilibrium states so the equations in (2a)-(2d) apply to both states.

(b) If total THC density is dropping, then model equation (1d) implies that the uninfected THC density regeneration rate has reached its maximum value $g_{\max }$, which will be assumed to be true here.

(c) It will be assumed that the viral load at the beginning and end of the THC drop leading to AIDS are identical. Thus, if the time of the beginning and end of the drop are denoted by $t_{\mathrm{e} 1}$ and $t_{\mathrm{e} 2}$, respectively, then it will be assumed that $V\left(t_{\mathrm{e} 1}\right)=V\left(t_{\mathrm{e} 2}\right)$, a value that will be called the critical viral load. Using (2a), this latter assumption results in

$$
\frac{d\left(t_{\mathrm{e} 1}\right) d^{*}\left(t_{\mathrm{e} 1}\right)}{p\left(t_{\mathrm{e} 1}\right)}=\frac{d\left(t_{\mathrm{e} 2}\right) d^{*}\left(t_{\mathrm{e} 2}\right)}{p\left(t_{\mathrm{e} 2}\right)} .
$$

(d) Since the infected THC density $T *(t)$ is a very small fraction of the total THC density $T^{+}(t)$ in the post acute infection phase period, the only way the total THC density in the final AIDS state can be a small fraction of what it was before the drop began is for the uninfected THC density to drop to a small percentage of its initial value. Using (3) 
above in (2c) gives

$$
\frac{p^{*}\left(t_{\mathrm{e} 2}\right)}{p^{*}\left(t_{\mathrm{e} 1}\right)}=\frac{T\left(t_{\mathrm{e} 1}\right)}{T(\mathrm{e} 2)},
$$

so that the only way for the ratio on the right to drop by 1-2 orders of magnitude is for the ratio on the left to decrease by $1-2$ orders of magnitude. Thus, the entire drop in the uninfected THC density leading to AIDS can be generated by a 1-2 order of magnitude increase in the infectivity rate parameter $p *(t)$.

(e) Extrapolating the result in (d) backwards in time leads to the conclusion that the quasi-static evolution of the viral load and infected THC density curves in HIV infection are generated by a relentless increase in the infectivity of CD4 + target cells during the incubation period. This evolution ends with a dangerous drop in the uninfected THC density and the development of chronic AIDS.

(f) The simulation of the drop in the uninfected THC density leading to chronic AIDS appears in the appendix. Since the simplest simulation of this drop is sought here, this simulation will assume that the model parameters $p, d$ and $d *$ are constants during the drop but the infectivity rate parameter $p^{*}(t)$ is time-dependent. Using the notation in (2a)-(2d), during the drop, this assumption leads to the requirement that $p\left(t_{\mathrm{e} 1}\right)=p\left(t_{\mathrm{e} 2}\right)=$ constant, $d\left(t_{\mathrm{e} 1}\right)=d\left(t_{\mathrm{e} 2}\right)=$ constant, and $d *\left(t_{\mathrm{e} 1}\right)=d *\left(t_{\mathrm{e} 2}\right)$ $=$ constant. This simplifying assumption is a special case of (3) and therefore will be compatible with the data in Ref. [7]. How realistic the assumptions in $(f)$ are in practice can only be ascertained from experiment, but there is no doubt that this simulation is theoretically possible. The consequences of this HIV model will be explored in the remaining part of this paper and the results compared with experimental data.

\section{The HIV-1 acute infection phase}

Data on three important HIV-1 infections in the acute phase were compiled by Sandro Vento et al. [8] and appear in the second column in table 1. The total THC densities of the three patients before they became infected centered around 1000 cells $/ \mathrm{mm}^{3}$, and these densities, following infection, dropped to below 100 cells $/ \mathrm{mm}^{3}$ (AIDS state levels) for what turned out to be brief periods of time (a matter of days). However, all three of these infecteds had Pneumocystis carinii pneumonia (PCP) at presentation, which is one of the AIDS defining disorders. Moreover, patient (3) came down with oral candidosis on day 11 after presentation, which is a characteristic disease of ARC. The total THC density of all three patients quickly rebounded to normal levels, and no other opportunistic infection characteristic of AIDS was developed by this cohort during this study.

Because all three members of this cohort at presentation satisfied two criteria of AIDS as defined by the Centers for Disease Control (CDC), the point of view to be taken in this paper is that all three members of this cohort lapsed into what will be called a transient AIDS state in the acute infection phase due to unusually severe (temporary) immunosuppression. Because this data is not commonly known within the AIDS research community, most researchers probably never considered the concept of a transient AIDS state in the acute infection phase of HIV infection. 
The model presented in section 2 above will now be used to show that the decline in the total THC density in the acute infection stage is brought about by a delayed but very strong $\mathrm{CD} 8+\mathrm{T}$ cell response to the HIV challenge so that the destruction rate of infected THCs exceeds the maximum regeneration rate of uninfected THCs for a limited period of time. This CD8+ T cell response is expressed in the model by an increasing value of the infected THC density destruction rate $d *(t)$.

Following inoculation, the immune system is caught off-guard and the viral load and the infected CD4 + cell density grow exponentially. Once the CD8+ T cell response to HIV builds up, the rate at which infected THC cells are destroyed greatly increases. Because of homeostatic constraints, the immune system responds to this loss by increasing the uninfected THC production rate $g(t)$. However, the value of the uninfected THC regeneration rate $g(t)$ has an upper limit for every host, so the regeneration function $g(t)$ in (1c) and (1d) has a maximum value to be denoted by $g_{\max }$.

Equation (1d) describes the key dynamical mechanism that explains the drop in the total THC density in the acute infection phase. Whenever the destruction rate of the infected THC pool exceeds the regeneration rate of the uninfected THC pool, the total THC pool must drop. There is nothing mysterious about this result, it merely states an obvious conservation law that can be derived from (1e) by simply differentiating both sides of (1e) with respect to time and using (1b)-(1d). Thus, during the drop in the total THC density in the acute infection stage, equation (1d) leads to

$$
T^{*}(t)=\frac{1}{d^{*}}\left[g_{\max }-\mathrm{d} T^{+}(t) / \mathrm{d} t\right] .
$$

This equation will be used to calculate the infected THC density $T *(t)$ in the acute infection stage from the data in table 1.

Now the maximum value of the THC regeneration rate has been measured. For a cohort of HIV-infected adults undergoing HAART, Stuart et al. [9] found that the average regeneration rate of naïve CD4+ T cells was $0.34 \pm 0.04$ cells $/ \mathrm{mm}^{3} /$ day although this value was mildly age dependent. Stuart also found that the maximum average regeneration rate of memory T cells was $1.46 \pm 0.4$ cells $/ \mathrm{mm}^{3} / \mathrm{day}$, a value that was not age dependent. Thus, the maximum average regeneration rate in the total THC density was found to be about $g_{1, \max }=1.80$ cells $/ \mathrm{mm}^{3} /$ day. The authors' experiments led them to conclude that the thymus plays an important role in naïve $\mathrm{T}$ cell regeneration.

Also, the average destruction rate of infected THCs was measured by Mittler et al. [10] during the steady-state phase of HIV infection, and it was found to be a constant equal to $d^{*}=0.5$ per day, with a relatively small standard deviation over the cohort.

To proceed with this calculation from the available data in table 1, it was assumed that the drop in the total THC density started mid-way between the time the pre-infection baseline measurement was made and the time of presentation. Obviously then, actual inoculation with HIV is presumed to have occurred several days before the midpoint in this time interval. The average value of $\mathrm{d} T^{+}(t) / \mathrm{d} t$, denoted by $\left\langle\mathrm{d} T^{+}(t) / \mathrm{d} t\right\rangle$, between two consecutive $T^{+}(t)$ measurement points was calculated and inserted into equation (5) to compute the average value of the infected THC density $T *(t)$, denoted by $\langle T *(t)\rangle$, over the time interval. It was further assumed that the slope $\mathrm{d} T^{+}(t) / \mathrm{d} t$ at the beginning of the drop and at the time PCP was developed in these three patients (the lowest recorded value of $T^{+}(t)$ ) was zero. The result of this calculation is shown in the fourth column in table 1. The average value of the total THC density $T^{+}(t)$, denoted by $\left\langle T^{+}(t)\right\rangle$, over the interval appears in column five in table 1 , and the 
calculated value of the average percentage of THCs that are infected with HIV is shown in the last column in table 1 .

Looking at the results in the last column in table 1, we see that at one time in the acute infection stage, between $20.5 \%$ and $40.0 \%$ of the total THCs of these patients were HIVinfected. These percentages may be unusually high for HIV infection in the acute stage, or these three patients may just have been unusually unlucky in being exposed to an AIDS defining antigen at the exact time when their immune systems were extremely vulnerable.

Data on an acute HIV infection case similar to those presented in Ref. [6] has been reported by Kaushal Gupta [11]. At presentation, the total THC density of this patient was $255 \mathrm{cell} / \mathrm{mm}^{3}$ and on its way up. At presentation the patient was found to have cytomegalovirus (CMV) colitis, which is an AIDS defining infection. Although it is very likely that the total THC density of this patient fell below 200 cells $/ \mathrm{mm}^{3}$ before presentation, there is no data to prove this. Thus, once again, it is possible to pass through a transient AIDS stage in the acute phase of the infection.

Experimental measurement of the fraction of THCs that are HIV-infected during the steady-state phase of the infection yields values between 0.0001-1\% [12]. Measurements of the infected percentage during the acute infection stage can yield values over $50 \%$, albeit infrequently. Indeed, the fractions computed in the last column in table 2 completely agree with these data in the literature.

What happens after the infected THC density $T *(t)$ peaks in the acute phase can easily be explained using (1b). Here, the factor $d *(t) T *(t)$ is greater than $p *(t) T(t) V(t)$ so that $T *(t)$ starts declining. The right hand sides of (1c) and (1d) become positive, $T(t)$ and $T^{+}(t)$ start increasing, and the immune system rebounds by pulling itself out of being in an immunosuppressed state. On closer inspection this rebound of the immune system is possible because the value of the infectivity rate parameter $p *(t)$ is relatively small in the acute stage so that the infected THC density $T *(t)$ keeps declining and uninfected THC density $T(t)$ keeps rising for an extended period of time.

The value of the infectivity rate parameter $p *(t)$ during the acute infection phase is probably not very different from its value during the steady-state phase that follows it. Since all parameters saturate at constant values during the steady-state phase, the infectivity rate parameter is very likely to be a constant throughout the first stage of HIV infection so that $p^{*}=p *\left(t_{\mathrm{e}}\right)$. Since the fraction of THCs that are infected during the steady state phase is less than $1 \%$ of the total, the value of $p *\left(t_{\mathrm{e}}\right)$ in (2c) must be relatively small so that $T\left(t_{\mathrm{e}}\right)$ is large enough to be within $1 \%$ of $T^{+}\left(t_{\mathrm{e}}\right)$. In later stages of HIV infection, the value of $T\left(t_{\mathrm{e}}\right)$ drops from what it was in the first stage, so the value of $p *\left(t_{\mathrm{e}}\right)$ must increase.

The relatively low value of the infectivity rate parameter $p *(t)$ in the acute infection stage will be shown to eventually rise by at least a factor of ten to end the incubation period with chronic AIDS.

Thus, the model mechanism presented here credibly explains why these three patients experienced precipitous, transient drops in their total THC density causing the development of a transient AIDS state in the acute phase of HIV infection. These results strongly suggest that the infected THC density is a much better measure of disease progression than viral load.

The data in table 1 is incompatible with the anti-diversity model of the cause of AIDS [13]. According to this model, a prolonged incubation period is required to develop AIDS after inoculation because AIDS is a result of a "slow but steady" increase in viral diversity, generated by mutations, which eventually reaches such an unmanageable level that the immune system essentially collapses. The anti-diversity model cannot explain how a drop in the total THC density can develop in the acute infection phase, when the diversity within a 
Table 2. WHO disease staging system for HIV infection and AIDS in adults and adolescents following the acute infection phase (1990) [16].

\begin{tabular}{|c|c|c|c|}
\hline Clinical stage 1 & Clinical stage 2 & Clinical stage 3 & Clinical stage 4 \\
\hline 1. Asymptomatic & $\begin{array}{l}\text { 3. Weight loss }<10 \% \text { of body } \\
\text { weight }\end{array}$ & $\begin{array}{l}\text { 7. Weight loss }>10 \% \text { of body } \\
\text { weight }\end{array}$ & 14. HIV wasting syndrome \\
\hline \multirow[t]{15}{*}{$\begin{array}{l}\text { 2. Generalized lymphade- } \\
\text { nopathy }\end{array}$} & $\begin{array}{l}\text { 4. Minor mucocutaneous manifes- } \\
\text { tations }\end{array}$ & $\begin{array}{l}\text { 8. Unexplained chronic diarr- } \\
\text { hoea }>1 \text { month }\end{array}$ & 15. Pneumocystic carnii pneumonia \\
\hline & $\begin{array}{l}\text { 5. Herpes zoster within the last } 5 \\
\text { years }\end{array}$ & $\begin{array}{l}\text { 9. Unexplained prolonged fever }>1 \\
\text { month }\end{array}$ & 16. Toxoplasmosis of the brain \\
\hline & $\begin{array}{l}\text { 6. Recurrent upper respiratory tract } \\
\text { infections }\end{array}$ & 10. Oral candi-diasis (thrush) & 17. Cryptosporidiosis with diarrhoea $>1$ month \\
\hline & & 11. Oral hairy leucoplakia & 18. Cryptococcosis, extrapulmonary \\
\hline & & 12. Pulmonary tuberculosis & $\begin{array}{l}\text { 19. Cytomegalovirus disease of an organ other than liver, spleen, } \\
\text { or lymph node }\end{array}$ \\
\hline & & 13. Severe bacterial infections & $\begin{array}{l}\text { 20. Herpes simplex virus infection, mucocu-taneous ( }>1 \text { month) } \\
\text { or visceral }\end{array}$ \\
\hline & & & 21. Progressive multifocal leucoencephalopathy \\
\hline & & & 22. Any disseminated endemic mycosis \\
\hline & & & 23. Candidiasis of esophagus, trachea, bronchi \\
\hline & & & 24. Atypical mycobacteriosis \\
\hline & & & 25. Non-typhoid Salmonella septicemia \\
\hline & & & 26. Extrapulmonary tuberculosis \\
\hline & & & 27. Lymphoma \\
\hline & & & 28. Karposi's sarcoma \\
\hline & & & 29. HIV encephalopathy \\
\hline
\end{tabular}


host is relatively small, nor how such a drop could be reversed, rebounding as the diversity increases. Thus, the anti-diversity model is at a loss to explain how a transient AIDS state could develop in the acute infection phase of the disease, which the data in table 1 proves is possible.

By contrast, the model presented here offers a credible explanation of the data in table 1 and is consistent with THC infected fraction data in the literature, as shown.

\section{HIV-1 incubation period curve}

The time between seroconversion and the development of AIDS is known as the incubation period of the disease. The distribution of incubation periods for a random cohort of infecteds is known as the IPDC and is expressed as the fraction of the cohort $A(t)$ that has developed chronic AIDS at an elapsed time $t$ after seroconversion.

A meta-analysis of 38 studies involving $13030 \mathrm{HIV}$-infected patients measured the first 13 years of the IPDC [14], and the results are shown in figure 1.

One of the outstanding mysteries of AIDS is the great disparity in values of the incubation period for a random cohort of HIV-infected patients. From the data in figure 1, about $0.6 \%$ infecteds come down with AIDS within a year of seroconvertion, but a larger percentage of infecteds show no sign of immune system deterioration after 13 years of infection. "What accounts for the broad disparity in the incubation period distribution curve?" and "what triggers the onset of AIDS?" are questions that will be addressed and answered here. The only immunological data to be modeled in this section is shown in figure 1 ; the modeling of this data will lead to remarkable conclusions that will be shown to agree with other clinical data on HIV infection.

A phenomenological least-squares fit to these 13 data points was sought by the family of distribution curves

$$
A_{n}(t)=\frac{\int_{0}^{t} t^{n} \mathrm{e}^{-b t} \mathrm{~d} t}{\int_{0}^{\infty} t^{n} \mathrm{e}^{-b t} \mathrm{~d} t}=1-\mathrm{e}^{-b t} \cdot \sum_{k=0}^{n-1} \frac{(b t)^{k}}{k !}, \text { where } n=1,2,3, \ldots
$$

which assumes that everyone infected with HIV-1 will eventually develop AIDS if left untreated (we assumed here that $A(\infty)=1$ ).

Radioactive nuclear decay of an unstable nuclide into a stable nuclide in one random transition is a special case of the family of curves in equation (6). Choosing $n=1$, equation (6) reduces to the distribution $A_{1}(t)=1-\mathrm{e}^{-\mathrm{bt}}$ which accurately fits the measured data describing the fraction of un-decayed radioactive nuclei of a given nuclide present at $t=0$ that has decayed by time $t$. Here, the half-life of the nuclide is given by $T_{1 / 2}=(1 / b) \ln (2)$. Since radioactive decay is a random event, no radioactive nucleus has any knowledge of its history or how long it has been in existence (its age).

The best fit to the data in Ref. [14] occurs for $n=3$ with $b=0.25135$ (years) $^{-1}$, and this fit is also shown in figure 1. Thus, an excellent fit to the HIV-1 IPDC is given by the function $A_{3}(t)=1-\mathrm{e}^{-b t}\left[1+b t(1 / 2)(b t)^{2}\right]$, and an extrapolation of this function into the region $t>13$ years is also shown in figure 1. Projecting this fitted curve into the future, it is predicted that the incubation periods of $12 \%$ of the population would exceed 20 years, $4 \%$ of the population would exceed 25 years and $0.3 \%$ would exceed 40 years.

What physical meaning can be attached to the fact that that the distribution function in (6) for $n=3$ yields such an excellent fit to the AIDS incubation period curve? As will now be 
shown, the distribution function in equation (6) has a very important physical meaning and reveals important features about HIV infection.

Suppose there are four ordered stages to HIV infection, each clinically discernable, distinguishable from the others by different clinically observable disorders. Stage 1 of HIV infection will be defined as the stage that immediately follows seroconversion. Suppose at time $t=0$ a very large cohort of $N_{0}$ people are in stage 1 of the infection. As time goes on a certain number of this cohort make the transition to stage 2 of the infection by a completely random process. In the same way, a member of stage 2 can make the transition to stage 3 by a second random process. Finally, a person in stage 3 makes the transition to the fourth and final stage of the infection (the AIDS stage) by a third random process. If $N_{1}(t), N_{2}(t), N_{3}(t)$ and $N_{4}(t)$ denote the number of infecteds in each stage of the infection at time $t$, then these quantities satisfy the following set of coupled differential equations:

$$
\begin{gathered}
\mathrm{d} N_{1}(t)=-k_{1} N_{1}(t), \\
\mathrm{d} N_{2}(t)=k_{1} N_{1}(t)-k_{2} N_{2}(t), \\
\mathrm{d} N_{3}(t)=k_{2} N_{2}(t)-k_{3} N_{3}(t), \\
\mathrm{d} N_{4}(t)=k_{3} N_{3}(t),
\end{gathered}
$$

where $k_{1}, k_{2}$ and $k_{3}$ are the transition constants. In fact $(7 \mathrm{a})-(7 \mathrm{~d})$ describe the radioactive decay of a mother nuclide into a final, stable nuclide through two intermediate radioactive daughter nuclides [15]. If we assume that the values of all three transition constants are equal

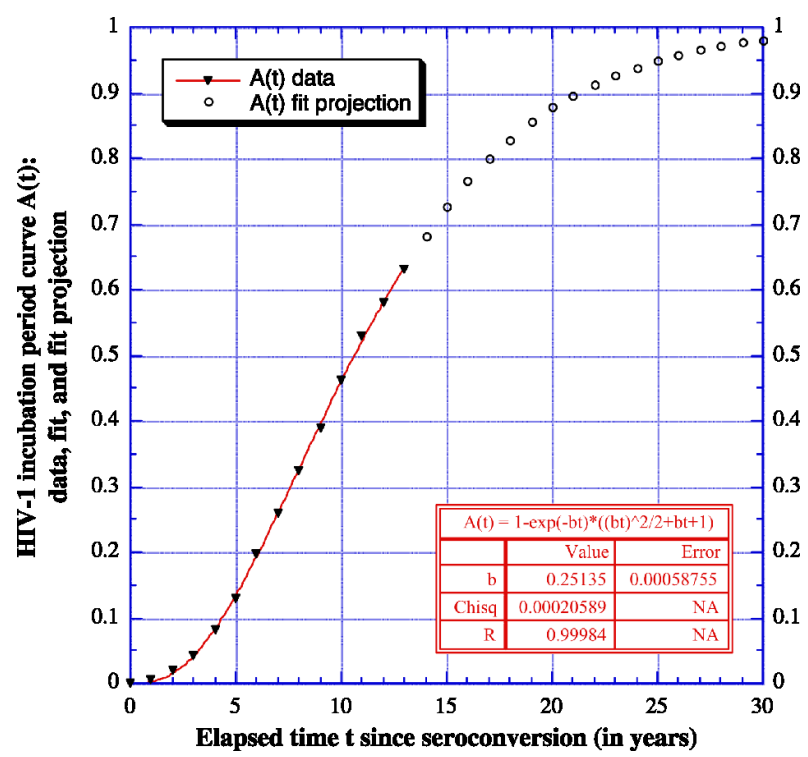

Figure 1. HIV-1 incubation period curve $A(t)$ : data [14], least squares fit, and fit projection. 
so that

$$
k_{1}=k_{2}=k_{3} \equiv b,
$$

then the solutions to (7a)-(7d) with the initial conditions of $N_{1}(0)=N_{0}, N_{2}(0)=0$, $N_{3}(0)=0$, and $N_{4}(0)=0$ can be put in the form of

$$
\begin{gathered}
H_{1}(t) \equiv \frac{N_{1}(t)}{N_{0}}=\mathrm{e}^{-b t}, \\
H_{2}(t) \equiv \frac{N_{2}(t)}{N_{0}}=b t \mathrm{e}^{-b t}, \\
H_{3}(t) \equiv \frac{N_{3}(t)}{N_{0}}=\frac{(b t)^{2}}{2} \mathrm{e}^{-b t},
\end{gathered}
$$

and

$$
H_{4}(t) \equiv \frac{N_{4}(t)}{N_{0}}=1-\mathrm{e}^{-b t} \cdot\left[1+b t+\frac{(b t)^{2}}{2}\right]=A_{3}(t) !
$$

The function in (9d) in the 3-transition model is precisely the function that results from fitting (6) to the IPDC data! Thus, the value of $n$ (a positive integer) represents the number of random transitions of the infection from seroconversion to the development of chronic AIDS. Since $n=3$ for HIV infection, the number of stages of the infection (equal to $n+1$ ) is 4 .

In the results in (9a) $-(9 \mathrm{~d}), H_{i}(t)$, where $i=1,2,3,4$, is simply the fraction of the cohort in stage $i$ at time $t$. Notice that $H_{1}(t)+H_{2}(t)+H_{3}(t)+H_{4}(t)=1$ as it must.

Thus, the modeling of the AIDS distribution curve above leads to the prediction that there are four distinct stages to HIV infection leading to chronic AIDS (the fourth and last stage of the infection) and the three transitions from one stage to the next have equal transition constants (see equation (8)). Thus, the development of AIDS after seroconversion is clearly a process, i.e. a result of an ordered sequence of three random transitions occurring with equal probability, and is not the result of a single event. Is this result biomedically credible?

Because CD4+ THC density testing in resource-poor countries is sometimes unavailable, the World Health Organization (WHO) has developed a staging system for HIV infection and AIDS in adults and adolescents that depends only on clinical manifestations of the disease [16]. The WHO staging is shown in table 2, and notice that there are 4 stages!

Stage 4 in table 2 is clearly the AIDS stage of HIV infection. Working backwards, stage 3 is clearly synonymous with what has been dubbed the ARC stage. Stage 2 is synonymous with what has been also called the early HIV symptomatic stage, and stage 1 begins immediately after seroconversion.

Thus, the 4 stages predicted by the modeling of the HIV incubation periods curve can be associated with the four clinical stages of the disease developed by the WHO shown in table 2. In the context of the disease model developed in this paper, each stage of the disease is associated with a mean value of the infectivity rate parameter $p *(t)$ that is less than the 
stage that comes after it. The value of the infectivity rate parameter in the fourth or last stage of the infection is large enough to cause AIDS.

What causes the infectivity rate parameter to increase in value from one stage to the next? The simplest possible answer to this question is to credit the increase in $p *(t)$ on random mutations of the virus into more infectious forms. Although the mutations that cause increases in the value of the infectivity rate parameter could require co-factors to occur, no such co-factor has been identified to date.

The value of the half-life of a nuclide is the statistical average of a large number of decays-individual nuclides have life-times that are different from this average in general. In the same way, individual infecteds may make the transition from one stage of HIV infection to the next at times that are different from the cohort average. However, transitions from one stage to the next in HIV infection in the model presented here are driven by random mutations of virions that increase the value of the viral infectivity over time in general.

Although the three transitions of HIV infection following seroconversion in the modeling here are mathematically isomorphic to a chain of three radioactive nuclear decays, there are two important differences between these two problems.

Firstly, all nuclei of a given nuclide are identical and indistinguishable, but members of a cohort of infecteds in a given stage of HIV infection are biomedically different and distinguishable in principle.

Secondly, the state of an HIV infection at any time for a given infected is described by the model constructed in this paper so that how close an infected is to a transition from one stage to the next can be estimated; no analogous estimation can be made for a nuclide. Thus, insofar as HIV infection is concerned, either the uninfected THC density $T\left(t_{\mathrm{e}}\right)$ or the infectivity rate parameter $p *\left(t_{\mathrm{e}}\right)$ (see equation (2c)) serves as a measure of disease progression; no analogous parameter exists for radioactive nuclear decay.

The modeling results obtained here also offer a ready explanation for the HIV long-term nonprogressors (LTNPs) intensely studied in the literature.

To distinguish the LTNPs from the progressors in stage 1 of the infection, the operational definition of LTNP to AIDS has been narrowed to include only those infecteds whose viral loads following seroconversion are below the measurable threshold density of 50 virions $/ \mathrm{cm}^{3}$; about $0.2-0.4 \%$ of the HIV-infected population falls into this group. In the context of the model presented here, however, it is not the value of the viral load or the infectivity rate parameter in stage 1 of the infection that is important but the rate at which the infectivity rate parameter is increasing in time. In the model analyzed here, stage 1 LTNPs are defined as those infecteds whose viral infectivity rate parameters $p *(t)$ change so slowly in time that these infecteds never make the transition to stage 2 regardless of the initial values of their viral loads. Thus, the slope of the infectivity rate parameter curve $p *(t)$ determines the duration of the HIV incubation period.

As we apply the modeling results obtained above, it must be remembered that these results apply only to cohort averages, not to individual cases. If the long-term nonprogressors are viewed as stuck in stage 1 of the infection, then (9a) gives the fraction of infecteds that are still in stage 1 as a function of time. Since $b=0.251$ (year) $^{-1}$, the time it takes for half of the infected cohort to make the transition to stage 2 is $T_{1 / 2}=\ln (2) /\left(0.251\right.$ year $\left.^{-1}\right)=2.76$ years. Thus, after 15 years the fraction of the original cohort that will still be stuck in stage 1 is $2^{-(15 / 2.76))}=0.0231=1 / 43.1$ on the average. Thus, $2.3 \%$ of HIV-infected patients are expect to be stuck in stage 1 of the infection 15 years after seroconversion. Proceeding in exactly the same way, $0.3 \%$ of infecteds will be found to be stuck in stage 1 of the infection 
23.1 years after seroconversion, and these are the LTNPs by the current definition; these results are certainly compatible with all the data in the literature on LTNPs. Thus, the novel modeling introduced here allows the fraction of infecteds who remain stuck in stage 1 of the infection at any time after seroconversion to be computed.

In exactly the same way, in a large sample of radioactive iodine-131 nuclei there will always be some that have not decayed even after an elapsed time of many half-lives. These long-term "non-decayers" are indistinguishable from the iodine-131 nuclei that subsequently decayed into xenon-131 with the emission of an electron. For example, since the half-life of iodine- 131 is 8.0197 days, $0.3 \%$ of a sample of iodine- 131 nuclei will still be present after an elapsed time of 67.21 days, equivalent to 8.380 half-lives. Thus, it is only by random chance that an iodine-131 nucleus is found not to have made the transition to xenon after an elapsed time equivalent to 8.38 half-lives.

Are LTNPs biomedically different from the progressors?

Clearly, the particular strains of HIV-1 infecting an individual do not determine the duration of the incubation period since identical strains of the virus infecting different individuals can produce very different incubation periods $[17,18]$. Certain co-infections also influence the rate of progression of HIV infection. Thus, genetic [19] and other factors are also important in determining the value of the incubation period for a particular individual. In conclusion, there is no absolute measure of the virulence of a viral strain.

Since progression of HIV infection depends on random mutation of HIV over time and cannot be predicted in advance for a given infected, and since there is no absolute measure of viral virulence, this model is compatible with the idea that there is no measurable biomedical difference between the LTNPs and the progressors. Indeed, no study to date has isolated any biomedical difference between the LTNPs and the progressors that can account for the different paths the disease takes in these two cohorts.

In addition to being able to be stuck in stage 1 for an inordinate amount of time it is also possible to be stuck in stage 2 in the same way for the same reason. The function $H_{1}(t)$ in equation (9b) describes the fraction of the entire original cohort of $N_{0}$ members that will be found in stage 2 as a function of the elapsed time $t$. This function reaches a maximum when $b t=1$, so that the maximum value of $H_{1}$ is $\mathrm{e}^{-1}=0.368$ and is reached at the time of $t_{\max }=b^{-1}=3.98$ years. At an elapsed time of 20 years, $H_{1}(20$ years $)=0.0329$ so $3.29 \%$ of the entire original cohort is in stage 2 , a significantly large percentage. As in stage 1 , by pure luck $50 \%$ of the infecteds in stage 2 at a certain time will still be in stage 2 after an elapsed time of 2.76 years, and $2.3 \%$ will still be in stage 2 after an elapsed time of 15 years. Thus, based on this random transition model, it would not be uncommon for an infected to be stuck in any stage of HIV infection for an inordinate amount of time, and these infecteds are biomedically no different from those who have made the transitions to the next stage on the average.

To complete this discussion, the function $H_{3}(t)$ in equation (9c) describes the fraction of the cohort that will be found in stage 3 as a function of the elapsed time $t$. This function reaches a maximum when $b t=\sqrt{2}$, so that the maximum value of $H_{3}$ is $\mathrm{e}^{-2}=0.135$ and is reached at the time of $t_{\max }=\sqrt{2} \cdot b^{-1}=5.63$ years. At an elapsed time of 20 years, $H_{3}(30$ years $)=0.0829$ so $8.29 \%$ of the entire original cohort is in stage 3 , again a significantly large percentage. As in previous stages, $50 \%$ of the infecteds in stage 3 at a certain time will still be in stage 3 after an elapsed time of 2.76 years, and $0.66 \%$ will still be in stage 3 after an elapsed time of 20 years-a small but significant percentage. A case like this exists in the literature: here, an infected who reports an occasional thrush, has never taken anti-virals, and has a CD4 + THC density of around 300 cells $/ \mu 1$ for 20 years with no further sign of immune 
system deterioration [20]. Referring to table (2), this infected is stuck in stage 3 of HIV infection by purely random luck.

Transitions between stages of HIV infection for a very large HIV-infected cohort occur randomly and are paradoxically not tied to the biomedical condition of infecteds on the average.

Finally in the limit when $b t \ll 1$, the function $A_{3}(t)$ behaves like $A_{3}(t) \Rightarrow(b t)^{3} / 3$ !. Thus, the very early incidence of AIDS for a cohort in stage 1 depends on the elapsed time cubed. For example, the fraction of the cohort acquiring AIDS at $t=6$ months is $2^{3}=8$ times greater than the fraction that comes down with AIDS at $t=3$ months.

The fact that HIV infection has 4 stages and that the three transitions from one stage to the next are isomorphic to the problem of a chain of radioactive nuclear decays is not only amazing but has important implications.

Firstly, in the model constructed in this paper, the evolution of HIV infection is driven by the increase in the infectivity rate parameter $p *(t)$. Since every HIV infection must pass through the 4 stages in sequence, the increase in $p *(t)$ over the course of, say, a day cannot be so large so that the patient jumps a stage (e.g. from stage 1 to 3 without passing through stage 2). From (2c) it is clear that as $p *\left(t_{\mathrm{e}}\right)$ increases, the uninfected THC density $T\left(t_{\mathrm{e}}\right)$ decreases. Moreover, since the infectivity rate parameter $p *(t)$ is proportional to the viral infectivity $\inf (t)$ discussed in section 2, each transition of HIV infection from one stage to the next is associated with a unique value of $T(t), p *(t)$ and $\inf (t)$ for any given infected. The values of the uninfected THC density $T(t)$ at the transition points are expected to vary from infected to infected. Thus, the infectivity rate parameter $p *(t)$ curve for any HIV infected must pass through points in each stage of HIV infection and in sequence.

Secondly, the fraction of the known infecteds in any stage of HIV infection that make the transition to the next stage per unit time is a constant whose value was found to be $b=0.2513$ (year) ${ }^{-1}$ independent of the stage. Another way to put this result of the modeling is to say that a time of $\ln (2) / b=2.76$ years is required for $50 \%$ of the known infecteds in any stage of HIV infection to make the transition to the next stage. Since the transition from one stage to the next is driven by increases in the infectivity rate parameter $p^{*}(t)$, and since changes in $p *(t)$ are generated by viral mutations, the same sort of random mutations are required in each stage of HIV infection to spontaneously induce the transition to the next stage.

The idea that mutations of HIV causes the increase in the infectivity rate parameter $p *(t)$ which drives the evolution of the infection is supported by experiment since Martinez et al. [21] have shown that the combination of strong HIV-1 specific CD4+ Th1 cell and IgG2 antibody responses is the best predictor for continued long term non-progression to AIDS.

If this model is correct, then there is no unique, special anti-viral substance secreted by the CD8+ T cells of LTNPs that accounts for their unusually long incubation periods; indeed, to date, no such substance has been found although researchers have sought this substance for years [22].

The points on the IPDC shown in figure 1 were generated before the advent of HAART that dramatically changed the ability to treat HIV infection. By dramatically reducing the viral load of a patient, HAART can bring the patient back to an earlier stage of infection for a (temporary) period of time. Thus, the stages of HIV infection are reversible in principle.

In the absence of any therapy, the fact that $0.6 \%$ of those who become infected with HIV-1 develop clinical AIDS in less than a year after seroconverting [14] proves that rapid progression to AIDS after seroconversion is possible. 
As has already been shown in the acute infection phase, when the immune system is incapable of producing enough uninfected CD4 + cells to replace the infected ones it is destroying, the THC density declines. When this happens at the end of stage 3 of the infection, the resulting hazardous decline in the total THC density inexorably leads to the development of chronic AIDS.

\section{The transition to chronic AIDS}

Plots of the THC density curve for five of the seven AIDS-transition cases measured by Saksela et al. [6] appear here in figure 2, with the curves for the remaining two cases looking very similar to these. Time $t=0$ in figure 2 corresponds to 12.01 AM on January 1, 1984 . The THC density curves of the remaining 11 study patients did not decline towards AIDS but fluctuated about mean equilibrium values very similar to the first 3 years of the five curves shown in figure 2. In fact, the Saksela et al. results imply that detectable levels of HIV-1 mRNA in an HIV-infected patient's PBMCs may be a necessary precursor to the subsequent development of AIDS.

The amount of HIV mRNA in the Saksela study was expressed in terms of the ratio of the number of copies of in vitro-transcribed control RNA molecules per microgram of PBMC RNA. A ratio below $1 \times 10^{3}$ copies/ $\mu$ g of PBMC RNA was undetectable, and a ratio above this threshold value was present in all seven patients throughout the period when their THC densities were collapsing from a normal value to one that that led to the development of AIDS. Interestingly, during this period of collapsing THC density leading to AIDS, the HIV mRNA ratio in these seven study subjects oscillated between a value in the $1-5 \times 10^{3}$ range to generally one in the $0.2-1 \times 10^{5}$ range.

Now just before the drop towards chronic AIDS begins, the last factor in equation (5) vanishes so that the value of the critical THC density is $T_{\mathrm{c}}^{*}=g_{\max } d^{-1}=3.6 \mathrm{cells} / \mu \mathrm{l}$. Since in the largely asymptomatic phase of HIV infection the total THC density must be greater than 500 cells/microliter, the beginning of the ARC stage of the infection, the percentage of THCs that are HIV infected in the asymptomatic phase must be less than $360 / 500=0.72 \%$, a very small percentage; for those with ARC or AIDS, this percentage must be greater than $0.72 \%$. Now experiment demonstrates that $0.0001-1 \%$ of THCs are HIV-producing during this disease [12], with the percentage for those with AIDS being about $2.5 \%$ on the average [7], results that agree with this model result. The fact that the number of infected THCs is on the order of 100 times higher in those with AIDS or ARC than in asymptomatic infecteds [7] also supports this model.

The appendix contains the complete, time-dependent solution to the transition to chronic AIDS using the model equations in (1a)-(1e) coupled with the AIDS transition model assumptions in section (4) above. The drop in the total THC density begins only after the viral load and the infected THC density reach their critical values at a time that will be taken to be $t=0$ for the sake of simplicity. Thus, in what follows, $V_{\mathrm{c}}=V(0)$ and $T_{\mathrm{c}}^{*}=T(0)$. Since times $t \leq 0$ describe a quasi-static equilibrium state, the equations in (2a) $-(2 d)$ lead to

$$
V_{\mathrm{c}}=\frac{p(0) g_{\max }}{d(0) d^{*}(0)}, \quad T_{c}^{*}=\frac{g_{\max }}{d^{*}(0)} \approx 3.6 \text { cells } / \mathrm{mm}^{3}, \quad \text { and } \quad T(0)=\frac{d(0) d^{*}(0)}{p(0) p^{*}(0)}
$$


The analysis in the appendix leads to the following results for the infected THC density $T *(t)$, the viral load $V(t)$, and the infectivity rate parameter $p *(t)$ during the drop in the total THC density $T^{+}(t)$ leading to AIDS:

$$
\begin{gathered}
\frac{T^{*}(t)}{T^{*}(0)}=\frac{V(t)}{V(0)}=1-\frac{1}{g_{\max }} \frac{\mathrm{d} T^{+}(t)}{\mathrm{d} t} \\
\frac{p^{*}(t)}{p^{*}(0)}=\frac{T(0)}{T(t)}
\end{gathered}
$$

As an example of the application of the results in (10a)-(10c), the data in Ref. [6] for patient B will be modeled. Connecting two consecutive data points on the total THC curve $T^{+}(t)$ by straight lines, the average values of $T^{+}(t)$ and $\mathrm{d} T^{+}(t) / \mathrm{d} t$ can be computed, and it will be assumed that these values apply to the mid-point of the time interval between these points. The resulting points on the $T^{+}(t)$ curve are plotted in figure 3 . Time $t=1$ year in figure 3 corresponds to $12.01 \mathrm{AM}$ on January 1,1985 . The results for $\mathrm{d} T^{+}(t) / \mathrm{d} t$ are inserted into (10b), along with $g_{\max }=1.8 \mathrm{cells} / \mathrm{mm}^{3} /$ day, to compute the ratios $T^{*}(t) / T^{*}(0)=V(t) / V(0)$, and the result is also plotted in figure 3; notice that these ratios never exceed the value of 3 .

Since $T *(0)=T_{\mathrm{c}}^{*}=3.6$ cells $/ \mathrm{mm}^{3}$, this last result can be used to compute the infected THC density curve $T *(t)$ during the drop, and the result also appears in figure 3; notice that the maximum value of $T *(t)$ during the drop is 11 cells $/ \mathrm{mm}^{3}$ so that $T *(t) \ll T^{+}(t)$ during the drop. The uninfected THC density $T(t)$ can now be computed from $T(t)=T^{+}(t)-T *(t)$, and since $T(0)=T^{+}(0)-T *(0)$ can also be computed, the ratio $p^{*}(t) / p *(0)=T(0) / T(t)$ can now be calculated; the result of this latter calculation is plotted in figure 3 . These results again

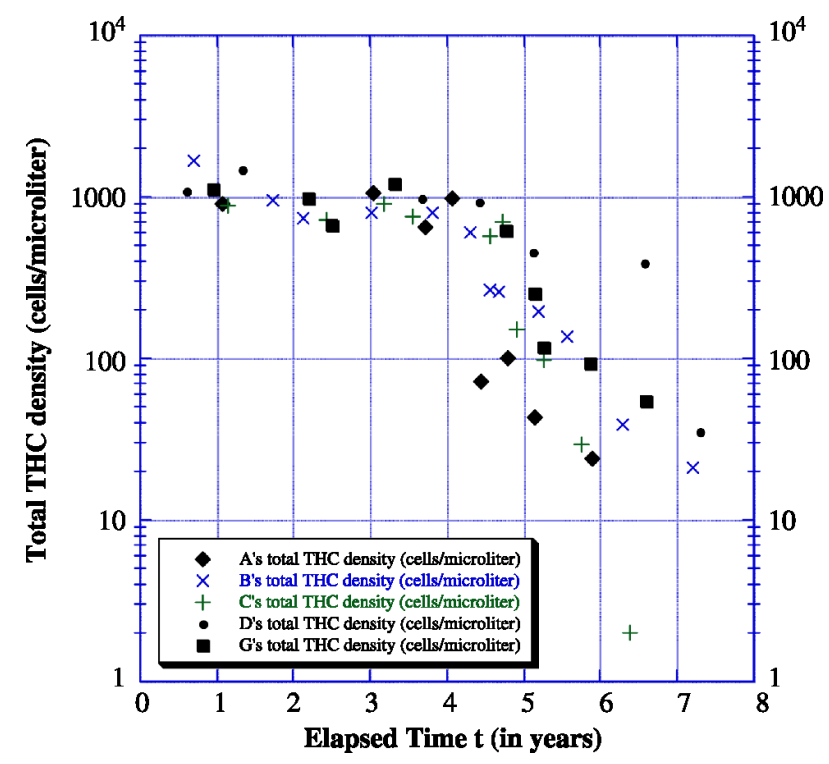

Figure 2. Total THC density curve $T^{+}(t)$ data during the transition to AIDS from Ref. [6]. 
strongly suggest that the infected THC density is a much better measure of disease progression than viral load.

It is clear from figure 3 that an increase in the infectivity rate parameter $p *(t)$ of target THCs is responsible for the triggering of the decline in the total THC density leading to AIDS. By the time Patient B's total THC density has declined to the value of $30 \mathrm{cells} / \mathrm{mm}^{3}$, the infectivity has increased by a factor of 64 times its value at the beginning of the drop.

Since $T^{+}(0)-T_{\mathrm{c}}^{*}=T(0)$, using (10a) leads to the following result for the critical value of the infectivity rate parameter $p *(0)$ that triggers the drop in $T^{+}(t)$ leading to AIDS:

$$
p_{\mathrm{c}}^{*} \equiv p^{*}(0)=\frac{g_{\max }}{V_{\mathrm{c}}\left[T^{+}(0)-T_{\mathrm{c}}^{*}\right]} .
$$

Since $T_{\mathrm{c}}^{*}=3.6 \mathrm{cells} / \mathrm{mm}^{3}$ and $g_{\max }=1.8$ cells $/ \mathrm{mm}^{3} /$ day, and since the patient's initial total THC density $T^{+}(0)$ can be measured, the value of $p *(0)$ can be computed from equation (11) if the critical viral load $V_{\mathrm{c}}$ for this patient were known. Since the viral set-point of HIV-infected patients typically varies from $10^{2}$ to $10^{4}$ virions $/ \mathrm{mm}^{3}$, and since the value of $T^{+}(0)$ can vary by at least a factor of 4 (from 500 to over 2000 cells $/ \mathrm{mm}^{3}$ ), it is clear that the value of the infectivity can vary by a factor of at least 100 . Thus, the increase of the infectivity by a factor of 64 for patient B shown in figure 3 is certainly feasible. Sixty-fold changes in viral infectivity have, in fact, been measured [24].

Since similar results are obtained for the other patients in Ref. [6], the model results for these patients were not included here.

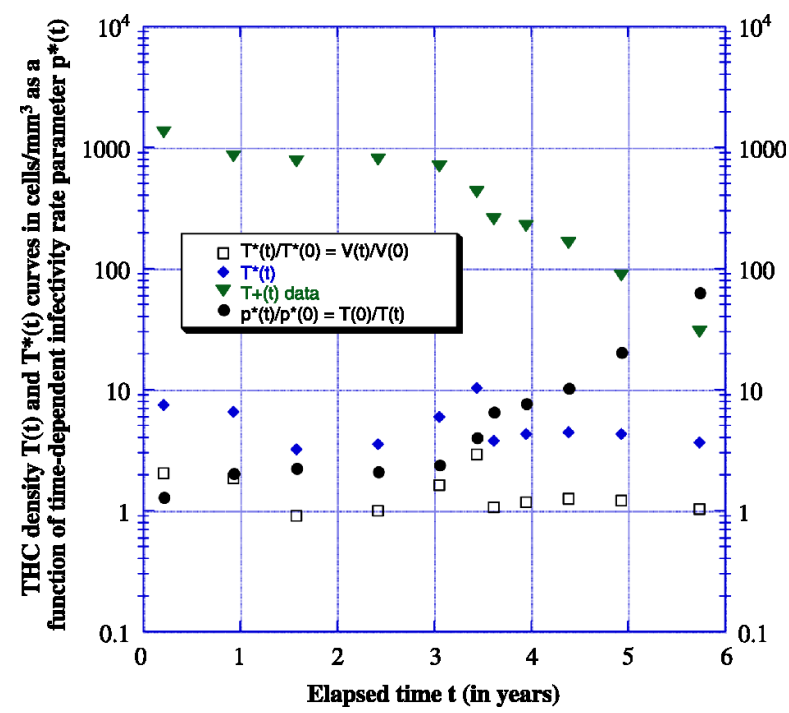

Figure 3. The transition to AIDS generated by a time-dependent infectivity rate parameter $p *(t)$ using patient B's total THC density $T^{+}(t)$ data from Ref. [6]. 


\section{HAART vs. alternative therapies}

The current major approach to controlling HIV-1 infection is HAART, a therapy that interferes with the ability of infected $\mathrm{CD} 4+$ cells to produce virulent virions. Although HAART has proven effective in controlling HIV infection for a limited period of time, it has not led to clearance or a cure of the disease.

The equations in (2a)-(2d) can be used to see the impact HAART therapy has on the steady-state infection. HAART generally reduces the value of the viral production parameter $p\left(t_{\mathrm{e}}\right)$, thereby reducing the value of the viral load in (2a) and increasing the value of the uninfected THC density in (2c). If the total THC density in (2d) is constant, then reducing the value of $p\left(t_{\mathrm{e}}\right)$, reduces the value of $g\left(t_{\mathrm{e}}\right)$ and keeps the infected THC density $T *(t)$ in (2b) below the critical value necessary to generate AIDS. These arguments are valid provided the infectivity rate parameter $p *(t)$ does not increase in time, which it unfortunately does. Thus, the increasing value of the infectivity would eventually undermine any fixed HAART therapy and would eventually lead to AIDS. Clearly then, the most direct way to prevent the development of AIDS is to reduce the infectivity rate parameter $p *\left(t_{\mathrm{e}}\right)$ to near zero indefinitely by the development of effective, well-tolerated, co-receptor blockers.

To date, HAART has failed to cure HIV infection because it has proven impossible to eliminate all infected CD4 + cells from a host (at the minimum, latently infected long-lived memory THCs remain). It must also be remembered that HAART cocktails eventually lose their potency over time as viral mutation renders them increasingly ineffective. Moreover, HAART has minimal impact on the pool of HIV-infected CD4+ natural killer (NK) cells that the immune system is dynamically incapable of eliminating [1,2]. It is also important to note that vaccine and IL-2 therapies to date have failed to strengthen significantly the immune system's defenses against HIV infection. Therefore, it is prudent to seek alternatives to HAART, vaccine, and IL-2 therapies if the goal is to develop an effective, durable treatment for HIV-1 infection.

It is important to remember that about $1 \%$ of Caucasians whose CD4+ cells lack coreceptor molecules, such as CCR5 and CXCR 4 cell surface receptors, cannot be infected by HIV-1 and are immune to this disease. On numbers alone, discovering a safe and effective fusion blocker that would prevent HIV-1 from binding to a known handful of co-receptor molecules on the surface of CD4 + cells would seem be an easier task than discovering a safe HAART that would be effective against all strains of a rapidly mutating virus. If an effective co-receptor blocker could be discovered, then administered as a prophylactic to uninfected people engaged in high-risk behavior, it would prevent infection, and administered to those already infected with HIV would prevent the transition to AIDS.

However, recent experiments by Nobuto Yamamoto and his research team on HIV-infected patients using an enzymatically modified serum vitamin $\mathrm{D}_{3}$-binding protein called $\mathrm{Gc}$ macrophage activating factor (GcMAF) stimulated macrophage action against HIV and eradicated all traces of viral antigens in the patients' blood for 2 years after the completion of therapy [23]. Thus, hope of finding a way to strengthen the immune response against HIV infection so that the fatal nature of the disease is eliminated should not be abandoned.

\section{Conclusion}

Dynamical modeling has been shown to be a powerful technique that can synthesize disparate data to answer some of the most fundamental questions about HIV infection. 
It has been demonstrated here that the inability of the immune system to produce enough uninfected THCs cells to replace the infected ones it is destroying leads to the drop in the total THC density at any stage of the infection. In the acute infection stage a transient drop in the total THC density can produce a transient AIDS state, lasting on the order of several days. Patients who went through a transient AIDS state in the acute phase of the infection were calculated to have had at least $20 \%$ of their total THCs infected with HIV at some time during this phase, a fraction that agrees with experimental data.

Modeling the IPDC led to the prediction that there are 4 stages to HIV infection following seroconversion, a prediction that exactly coincides with the WHO's identification of four clinical stages to the infection following the acute infection phase. The fraction of any cohort in any stage of the infection that makes the transition to the next stage per unit time was found to be 0.2513 per year. The model not only predicts long term non-progressors to HIV infection but predicts that $0.3 \%$ of infecteds will remain stuck in stage 1 of the infection for at least 23.1 years after seroconversion.

The transition from one stage to the next is driven by random events, identified with the random mutation of HIV into more infectious strains. The model presented here is compatible with there being no biomedical identifiable difference between the LTNPs and the progressors to HIV infection; all LTNP are simply regarded as lucky since their viral infectivities increase very slowly or not at all in time.

In the quasi-static, steady-state phase of infection a precipitous drop in the THC density by a factor of 10-100 causes chronic AIDS to develop and signals the end of the incubation period. In the modeling presented here, this drop in the THC density is traced to a 10-100fold increase in the infectivity of THC target cells, probably caused by viral mutation. In the modeling presented here, the essential difference between the transient AIDS state in the acute phase of the infection and the chronic AIDS state at the end of the incubation period is a $10-100$-fold increase in viral infectivity by the time chronic AIDS is developed.

The modeling results strongly suggest that the infected THC density is a much better measure of disease progression than viral load. As shown by the modeling, to prevent the development of chronic AIDS, the infected THC density must be kept below a critical value $T_{\mathrm{c}}^{*}=g_{\max } / d^{*}\left(t_{\mathrm{e}}\right)$, equal to 3.6 cells $/ \mathrm{mm}^{3}$ on the average. Thus, the most direct way to prevent chronic AIDS in HIV infection is to keep the number of infected THCs as small as possible by any means necessary.

\section{References}

[1] Valentin, A., Rosati, M., Patenaude, D.J., Hatzakis, A., Kostrikis, L.G., Lazanas, M., Wyvill, K.M., Yarchoan, R. and Pavlakis, G.N., 2002, Persistent HIV-1 infection of natural killer cells in patients receiving highly active antiretroviral therapy, PNAS, 99(10), 7015-7020, May 14.

[2] Antonio, V. and Pavlakis, G.N., 2003, Natural killer cells are persistently infected and resistant to direct killing by HIV-1, Anticancer Research, 23(3A), 2071-2075, May-June.

[3] Valentin, A., Trivedi, H., Lu, W., Kostrikis, L.G. and Pavlakis, G.N., 2000, CXCR4 mediates entry and productive infection of syncytia-inducing (X4) HIV-1 strains in primary macrophages, Virology, 269, 294-304.

[4] Perelson, A.S., Neumann, A.U., Markowitz, M., Leonard, J.M. and Ho, D.D., 1996, HIV-1 dynamics in vivo: virion clearance rate, infected cell life-span, and viral generation time, Science, 271(15), 1582-1586, March.

[5] Kramer, I., 2006, Understanding HIV infection through dynamical modelling: why preventative vaccines, therapeutic vaccines, and IL-2 therapy are not working, Mathematical and Computer Modelling, 44, 577-594.

[6] Saksela, K., Stevens, C., Rubinstein, P. and Baltimore, D., 1994, Human immunodeficiency virus type $1 \mathrm{mRNA}$ expression in peripheral blood cells predicts disease progression independently of the numbers of CD4+ lymphocytes, Proceedings of the National Academy of Sciences USA, 91, 1104-1108, February.

[7] Ho, D.D., Moudgil, T. and Masud, A., 1989, Quantitation of human immunodeficiency virus type 1 in the blood of infected persons, New England Journal of Medicine, 321(24), 1621-1625. 
[8] Sandro, V. and Giovanni, D.P., 1993, Pneumocystis carinii pneumonia during primary HIV-1 infection, Lancet, 342(8862), 24-26.

[9] Cohen Stuart, J, Hamann, D., Borleffs, J., Roos, M., Miedema, F., Boucher, C. and de Boer, R., 2002, Reconstitution of naïve $\mathrm{T}$ cells during antiretroviral treatment of HIV-infected adults is dependent on age, AIDS, 16(17), 2263-2266, November 22.

[10] Mittler, J.E., Markowitz, M., Ho, D.D. and Perelson, A.S., 1999, Perelson, improved estimates for HIV-1 clearance rate and intracellular delay, AIDS, 13(11), 1415-1417.

[11] Gupta, K.K., 1993, Acute Immunosuppression with HIV seroconversion, New England Journal of Medicine, 326(4), 288-289, January 28.

[12] Gupta, P., Kingsley, L., Armstrong, J., Bing, M., Cottrill, M. and Rinaldo, C., 1993, Enhanced expression of human immunodeficiency virus type 1 correlates with development of AIDS, Virology, 196, 586-595.

[13] Nowak, M.A., Anderson, R.M., McLean, A.R., Wolfs, T.F., Goudsmit, J. and May, R.M., 1991, Antigenic diversity thresholds and the development of AIDS, Science, 254(5034), 963-969, November 15.

[14] Time from HIV-1 seroconversion to AIDS and death before widespread use of highly-active antiretroviral therapy: a collaborative re-analysis, Collaborative Group on AIDS Incubation and HIV Survival including the CASCADE EU Concerted Action, 2000, Lancet, 355, 1131-1137.

[15] Kaplan, I., 1962, See, for Example, Nuclear Physics, 2nd ed. (Glen View, IL: Addison-Wesley), pp. $239-244$.

[16] The Different Stages of HIV Infection, June 26, 2002, Avert. Feb. 8, 2004 [http://www.avert.org/hivstages.html]

[17] Blick, G., Grieger-Zanlunge, P., Heseltine, et al., 2005, Patient zero: the connecticut source of the multidrug resistant, dual tropic, rapidly progressing strain found in New York city, Program and abstracts of the $3^{\text {rd }}$ IAS Conference on HIV Pathogenesis and Treatment; July 24-27 ; Rio de Janeiro, Brazil. Abstract $\mathrm{MoOa0101}$

[18] Markowitz, M., Mohri, J., Mehandru, S., et al., 2005, Infection with multi-drug resistant, dual-tropic HIV-1 and rapid progression to AIDS; a case report, Lancet, 365, 1031-1038.

[19] Migueles, S.A., et al., 2000, HLA B*5701 is highly associated with restriction of virus replication in a subgroup of HIV long term nonprogressors, Proceedings of the National Academy of Sciences USA, 97(6), 2709-2714, March.

[20] Milano, M., Wondering aloud: theories of one (Quasi) LTNP, ACRIA Update, Winter 2001/02-Vol. 11, No. 1

[21] Valerie, M., Dominique, C., Olivia, B., Nicole, N'go, Aurelie, S., Ioannis, T., Jean-Pierre, C, Didier, S., Henrie, A., Patrice, D., Christine, R. and Brigitte, A., 2005, Combination of HIV-1 specific CD4 TH1 cell responses and IgG2 antibodies is the best predictor for persistence of long-term nonprogression, The Journal of Infectious Diseases, 191(12), 2053-2063, Epub 2005 May 10.

[22] Levy, J.A., 2003, The search for the CD8 + cell anti-HIV factor (CAF), Trends in Immunology, 24(12), 628-632, December.

[23] Yamamoto, N., 2006, Pathogenic significance of $(\alpha-\mathrm{N}$-acetylgalactosaminidase activity found in envelope glycoprotein gp160 of human immunodeficiency virus type 1, AIDS Research and Human Retroviruses, 22(3), $262-271$.

[24] Rousso, I., Mixon, M.B., Chen, B.K. and Kim, P.S., 2000, Palmitoylation of the HIV-1 envelope glycoprotein is critical for viral infectivity, PNAS, 97(25), 13523, December 5.

\section{A. Appendix}

\section{A.1 Dynamically modeling the drop in the CD4 + cell density leading to chronic AIDS}

The AIDS transition model assumptions (a)-(f) in section (4) above will now be used together with the model equations in (1a)-(1e) to describe the transition from the asymptomatic phase of HIV infection to the AIDS phase.

It should be emphasized that the viral load and the infected, uninfected, and total CD4+ cell densities during the drop are not given by the steady-state functions in (2a)-(2d) because these quantities are changing in time and are not in a steady-state (except at the beginning and end of the drop).

Accordingly, using equation (2b), the infected THC density during the drop will be set equal to:

$$
T^{*}(t)=\frac{g_{\max }}{d^{*}\left(t_{\mathrm{e} 1}\right)}+\frac{\mathrm{d} \rho(t)}{\mathrm{d} t} \equiv T_{\mathrm{c}}^{*}+\dot{\rho}(t)
$$


where $\rho(t)$ is a new function introduced here that will parametize the drop, and where $T_{\mathrm{c}}^{*} \equiv\left(g_{\max }\right) / d^{*}\left(t_{\mathrm{e} 1}\right)$ is the critical value of the CD4 + cell density. Here, the notation $\mathrm{d} \rho(t) / \mathrm{d} t \equiv \dot{\rho}(t), d^{2} \rho(t) / \mathrm{d} t^{2} \equiv \ddot{\rho}(t)$, etc. will be used. Since $T *(t)$ and its derivative must be continuous at $t=t_{\mathrm{e} 1}$ and $t=t_{\mathrm{e} 2}$, the function $\rho(t)$ must satisfy $\dot{\rho}\left(t_{\mathrm{e} 1}\right)=\ddot{\rho}\left(t_{\mathrm{e} 1}\right)=0$ as well as $\ddot{\rho}\left(t_{\mathrm{e} 1}\right)=0$.

Inserting equation (A1) into equation (1d) then gives

$$
\dot{\rho}(t)=-\frac{1}{d^{*}\left(t_{\mathrm{e} 1}\right)} \frac{\mathrm{d} T^{+}(t)}{\mathrm{d} t},
$$

where the right-hand side of equation (A2) can be determined from experiment. Thus, the function $\dot{\rho}(t)$ can be computed from (A2) and inserted into (A1) to compute $T^{*}(t)$. Notice that when $d T^{+}(t) / \mathrm{d} t=0, T *(t)$ becomes a constant.

Since equation (A2) begins and ends at equilibrium states, the right-hand side of equation (A2) must vanish at times $t=t_{\mathrm{e} 1}$ and $t=t_{\mathrm{e} 2}$ in order for $\mathrm{d} T^{+}(t) / \mathrm{d} t$ to be continuous at these times. Thus, $\dot{\rho}(t)$ must also vanish at $t=t_{\mathrm{e} 2}$ so that the result in equation (A1) gives

$$
T^{*}\left(t_{\mathrm{e} 1}\right)=\frac{g_{\max }}{d^{*}\left(t_{\mathrm{e} 1}\right)}=T^{*}\left(t_{\mathrm{e} 2}\right)=\frac{g_{\max }}{d^{*}\left(t_{\mathrm{e} 2}\right)} .
$$

Thus, the infected $\mathrm{CD} 4+$ cell density at the beginning and end of the drop are identical, and $d^{*}\left(t_{\mathrm{e} 1}\right)=d^{*}\left(t_{\mathrm{e} 2}\right)$.

In summary then, continuity of $T *(t)$ and $T^{+}(t)$ and their derivatives requires the boundary conditions:

$$
\frac{\mathrm{d} \rho(t)}{\mathrm{d} t} \equiv \dot{\rho}(t)=0 \quad \text { and } \quad \frac{d^{2} \rho(t)}{\mathrm{d} t^{2}} \equiv \ddot{\rho}(t)=0 \text { at } t=t_{\mathrm{e} 1} \text { and } t_{\mathrm{e} 2}
$$

Thus, $\rho(t)$ parametizes the deviation of the infected CD4+ cell density $T^{*}(t)$ from its equilibrium value during the drop.

Parenthetically, the integration of equation (A2) leads to

$$
T^{+}(t)-T^{+}\left(t_{\mathrm{e} 1}\right)=-d^{*}\left(t_{\mathrm{e} 1}\right)\left[\rho(t)-\rho\left(t_{\mathrm{e} 1}\right)\right] .
$$

Since $d^{*}\left(t_{\mathrm{e} 1}\right)$ and the function $T^{+}(t)$ has been measured in the literature, equation (A5) can be used to completely determine $\rho(t)$. Because of the result in (A5), the area under the $\dot{\rho}(t)$ curve from $t=t_{\mathrm{e} 1}$ to $t=t_{\mathrm{e} 2}, \rho\left(t_{e 2}\right)-\rho\left(t_{e 1}\right)$, is related to the total, overall drop in the total CD4+ density.

As with the infected CD4+ cell density $T *(t)$, the viral load $V(t)$ at the beginning and end of the drop will be assumed to be identical. Accordingly, using equation (2a), the viral load $V(t)$ during the drop is parametized by setting

$$
V(t)=V_{\mathrm{c}}+\dot{\sigma}(t), \quad \text { where } V_{\mathrm{c}} \equiv \frac{p\left(t_{\mathrm{e} 1}\right) g_{\max }}{d\left(t_{\mathrm{e} 1}\right) d^{*}\left(t_{\mathrm{e} 1}\right)} .
$$

Here, $V_{\mathrm{c}}$ is the critical viral load, and the newly introduced function $\dot{\sigma}(t)$ will soon be shown to be related to $\dot{\rho}(t)$ defined in equation (A1). Since the viral load and its derivative must be continuous at $t=t_{\mathrm{e} 1}$ and $t=t_{\mathrm{e} 2}$, the function $\sigma(t)$ introduced in (A6) must satisfy $\dot{\sigma}\left(t_{e 1}\right)=\ddot{\sigma}\left(t_{e 1}\right)=\dot{\sigma}\left(t_{e 2}\right)=\ddot{\sigma}\left(t_{e 2}\right)=0$, the same boundary conditions as $\rho(t)$ [see equation (A4)]. 
Using (A1), (A2) and (A6) in equation (1a) gives the differential equation

$$
\ddot{\sigma}(t)+d\left(t_{\mathrm{e} 1}\right) \cdot \dot{\sigma}(t)=p\left(t_{\mathrm{e} 1}\right) \cdot \dot{\rho}(t)=-\frac{p\left(t_{\mathrm{e} 1}\right)}{d^{*}\left(t_{\mathrm{e} 1}\right)} \frac{\mathrm{d} T^{+}(t)}{\mathrm{d} t}=-\frac{V_{\mathrm{c}} \cdot d^{*}\left(t_{\mathrm{e} 1}\right)}{g_{\max }} \frac{\mathrm{d} T^{+}(t)}{\mathrm{d} t} .
$$

The right-hand side of (A7a) consists of factors that have been measured in the literature; thus, the right-hand side of (A7a) can be experimentally determined, and (A7a) can then be used to solve for $\sigma(t)$ in a straight-forward way. An infinite series solution to (A7a) is

$$
\dot{\sigma}(t)=-\frac{p\left(t_{\mathrm{e} 1}\right)}{d\left(t_{\mathrm{e} 1}\right) d^{*}\left(t_{\mathrm{e} 1}\right)} \sum_{n=1}^{\infty} \frac{1}{\left[-d\left(t_{\mathrm{e} 1}\right)\right]^{(n-1)}} \frac{\mathrm{d}^{n} T^{+}(t)}{\mathrm{d} t^{n}} .
$$

Thus, if $T^{+}(t)$ is a constant, $\dot{\sigma}(t)=0$. From (A2) and (A7b) both $\dot{\rho}(t)$ and $\dot{\sigma}(t)$ are clearly functions of $\mathrm{d} T^{+}(t) / \mathrm{d} t$.

Using equations (A1) and (A6) in equation (1b) gives

$$
p^{*}(t) T(t)=\frac{\left[\dot{T}(t)+d^{*}\left(t_{\mathrm{e} 1}\right) T^{*}(t)\right]}{V(t)}=\frac{\left[\ddot{p}(t)+d^{*}\left(t_{\mathrm{e} 1}\right) T_{\mathrm{c}}^{*}+d^{*}\left(t_{\mathrm{e} 1}\right) \dot{p}(t)\right]}{\left[V_{\mathrm{c}}+\dot{\sigma}(t)\right]} .
$$

Since $\rho(t)$ and $\sigma(t)$ are known functions of $\mathrm{d} T^{+}(t) / \mathrm{d} t($ see (A2) and (A7b)), the last expression in (A8) can be reduced to something very simple. If $|\ddot{\rho}(t)| \ll d^{*}\left(t_{\mathrm{e} 1}\right)|\dot{\rho}(t)|$ or, equivalently, if

$$
\left|\frac{1}{d^{*}\left(t_{\mathrm{e} 1}\right)} \frac{\mathrm{d}^{2} T^{+}(t)}{\mathrm{d} t^{2}}\right| \ll\left|-\frac{\mathrm{d} T^{+}(t)}{\mathrm{d} t}\right|
$$

then the $\ddot{\rho}(t)$ term in the numerator on the right hand side of (A8) can be neglected. This inequality is certainly satisfied if we connect every pair of consecutive data points on the $T^{+}(t)$ curve by straight lines so that the $T^{+}(t)$ curve is made up of straight-line segments. Then $\mathrm{d}^{2} T^{+}(t) / \mathrm{d} t^{2}=0$ at every point between two consecutive data points so that $\ddot{\rho}(t)=0$. The same inequality in (A9) also guarantees that the expression for $\dot{\sigma}(t)$ in (A7b) can be approximated by the first term on the right hand side. Since experiment shows that $d\left(t_{\mathrm{e} 1}\right)$ $>d^{*}\left(t_{\mathrm{e} 1}\right)$ in general, the inequality in (A9) also leads to

$$
\dot{\sigma}(t) \approx-\frac{p\left(t_{\mathrm{e} 1}\right)}{d\left(t_{\mathrm{e} 1}\right) d^{*}\left(t_{\mathrm{e} 1}\right)} \frac{\mathrm{d} T^{+}(t)}{\mathrm{d} t} .
$$

Since the instantaneous value of $\mathrm{d} T^{+}(t) / \mathrm{d} t$ at the mid-point between two consecutive data points is, of course, unknown, connecting two consecutive data points by straight lines in the above discussion and using the average value of the slope over the interval in place of the instantaneous value (unknown) is the best that can be done under the circumstances. This practice is very common in the literature when the instantaneous slope of a function over an interval was not measured. Although the value of the error incurred by this approximation cannot be exactly quantified since the actual behavior of $T^{+}(t)$ between two consecutive data pints is unknown, the error is expected to be small enough so that it will not affect the broad conclusions of the modeling. The latter is certainly the case when the time between two consecutive data points is very small or when the change in $T^{+}(t)$ between two consecutive measurements is small with respect to the value of $T^{+}(t)$ itself. 
Thus, if (A9) is true, then (A8) becomes

$$
p^{*}(t) T(t) \approx \frac{\mathrm{d}^{*}\left(t_{\mathrm{e} 1}\right)\left[T_{c}^{*}-\frac{1}{\mathrm{~d}^{*}\left(t_{\mathrm{e} 1}\right)} \frac{\mathrm{d} T^{+}(t)}{\mathrm{d} t}\right]}{\frac{p\left(t_{\mathrm{e}}\right)}{d\left(t_{\mathrm{e} 1}\right)}\left[\frac{d\left(t_{\mathrm{e} 1}\right)}{p\left(t_{\mathrm{e} 1}\right)} V_{\mathrm{c}}-\frac{1}{\mathrm{~d}^{*}\left(t_{\mathrm{e} 1}\right)} \frac{\mathrm{d} T^{+}(t)}{\mathrm{d} t}\right]}=\frac{d\left(t_{\mathrm{e} 1}\right) d^{*}\left(t_{\mathrm{e} 1}\right)}{p\left(t_{\mathrm{e} 1}\right)}=p^{*}\left(t_{\mathrm{e} 1}\right) T\left(t_{\mathrm{e} 1}\right)=\mathrm{constant}
$$

since $\left(d\left(t_{\mathrm{e} 1}\right) V_{\mathrm{c}}\right) /\left(p\left(t_{\mathrm{e} 1}\right)\right)=T_{\mathrm{c}}^{*}$. Thus, for example, a ten-fold increase in the infectivity rate parameter leads to a ten-fold drop in the uninfected THC density and a similar drop in the total THC density.

Using (1e), (A1), (A2) and (A11), the equation relating $T^{+}(t)$ to $p^{*}(t)$ is

$$
T^{+}(t)+\frac{1}{\mathrm{~d}^{*}\left(t_{\mathrm{e} 1}\right)} \frac{\mathrm{d} T^{+}(t)}{\mathrm{d} t}=\frac{d\left(t_{\mathrm{e} 1}\right) \mathrm{d}^{*}\left(t_{\mathrm{e} 1}\right)}{p\left(t_{\mathrm{e} 1}\right)} \frac{1}{p^{*}(t)}+T_{\mathrm{c}}^{*} \equiv F(t) .
$$

The infinite series solution to (A12a) is

$$
T^{+}(t)=F(t)+\sum_{n=1}^{\infty} \frac{1}{\left[-\mathrm{d}^{*}\left(t_{\mathrm{e} 1}\right)\right]^{n}} \frac{\mathrm{d}^{n} F(t)}{\mathrm{d} t^{n}}
$$

so that $T^{+}(t)$ is a function of $p^{*}(t)$ and its derivatives. Thus, both $\dot{\rho}(t)$ and $\dot{\sigma}(t)$ are functions of the time-derivatives of the inverse of the infectivity rate parameter $p^{*}(t)$. From (A1) and (A6) this means that both $T^{*}(t)$ and $V(t)$ during the drop are functions of time-derivatives of the inverse of the infectivity rate parameter $p^{*}(t)$; in particular, if $p *(t)$ is a constant, then so is $T^{+}(t)$. 


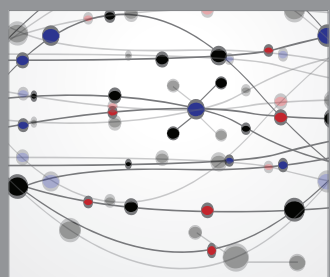

The Scientific World Journal
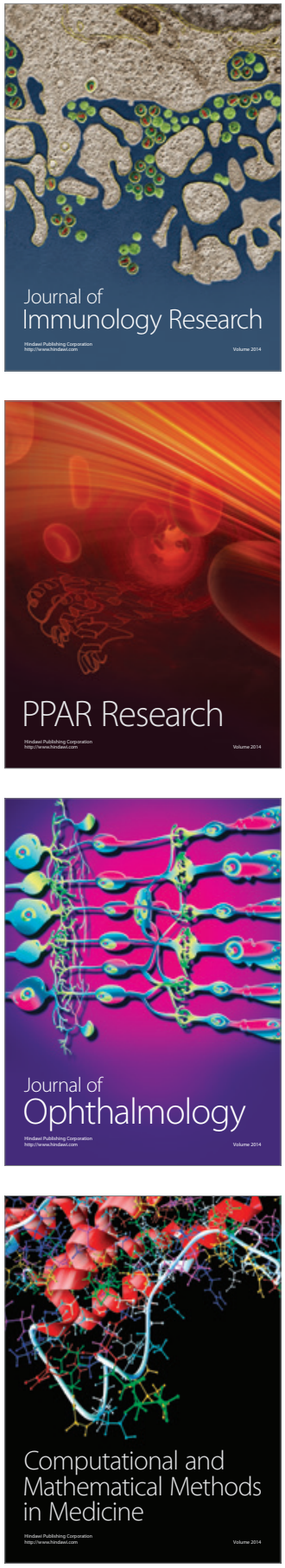

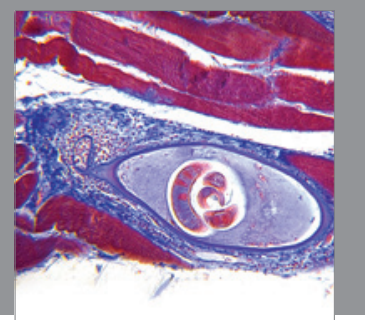

Gastroenterology

Research and Practice
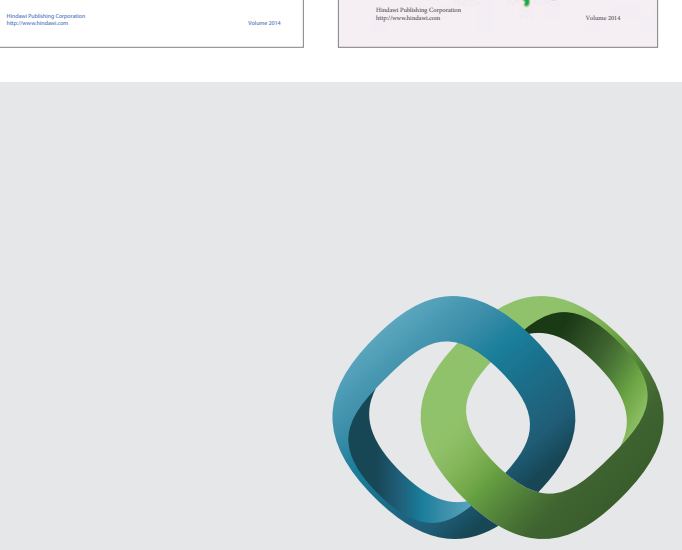

\section{Hindawi}

Submit your manuscripts at

http://www.hindawi.com
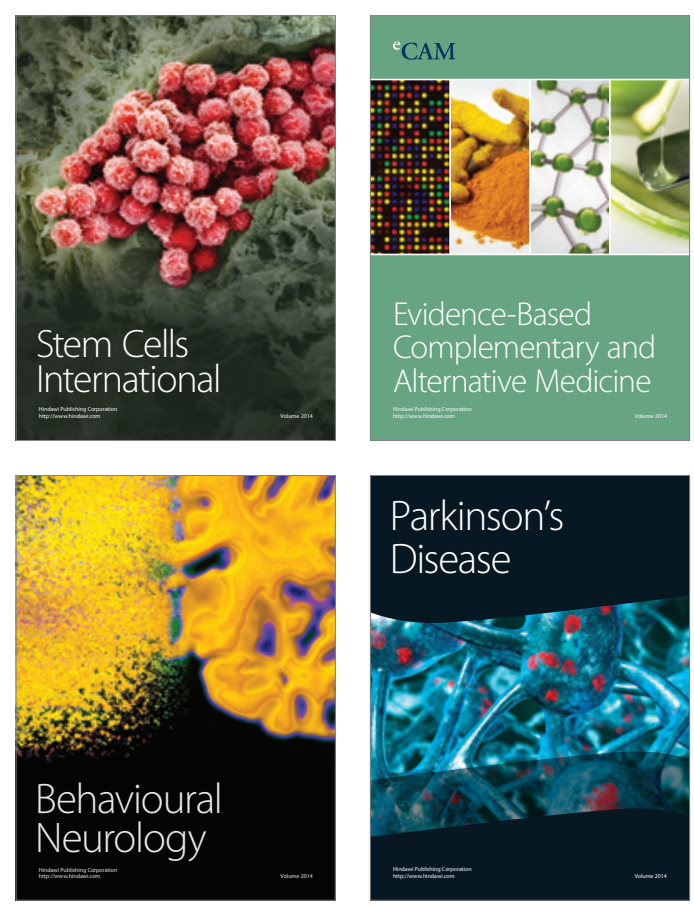

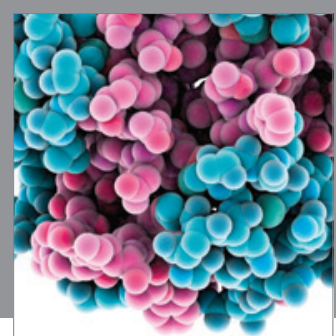

Journal of
Diabetes Research

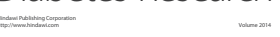

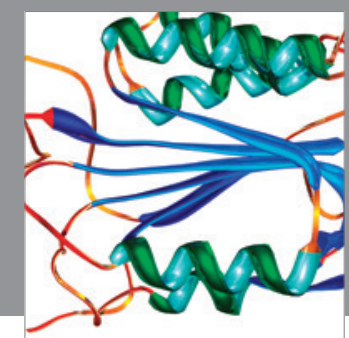

Disease Markers
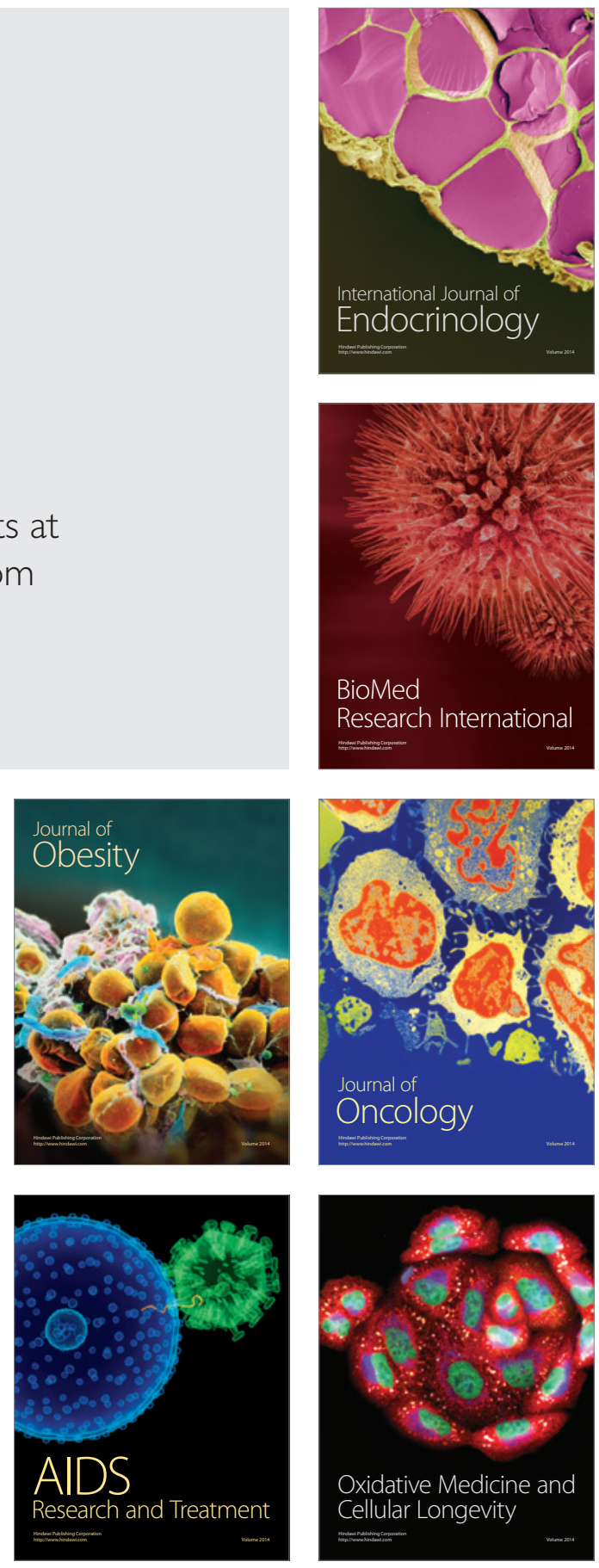